\title{
ALGEBRAIC STRUCTURE OF COMBINED TRACES *
}

\author{
ŁUKASZ MIKULSKI
}

Faculty of Mathematics and Computer Science, Nicolaus Copernicus University, Torun, Chopina $12 / 18$, Poland, and

School of Computing Science, Newcastle University, Newcastle upon Tyne, NE1 7RU, U.K. e-mail address: lukasz.mikulski@mat.umk.pl

\begin{abstract}
Traces - and their extension called combined traces (comtraces) - are two formal models used in the analysis and verification of concurrent systems. Both models are based on concepts originating in the theory of formal languages, and they are able to capture the notions of causality and simultaneity of atomic actions which take place during the process of a system's operation. The aim of this paper is a transfer to the domain of comtraces and developing of some fundamental notions, which proved to be successful in the theory of traces. In particular, we introduce and then apply the notion of indivisible steps, the lexicographical canonical form of comtraces, as well as the representation of a comtrace utilising its linear projections to binary action subalphabets. We also provide two algorithms related to the new notions. Using them, one can solve, in an efficient way, the problem of step sequence equivalence in the context of comtraces. One may view our results as a first step towards the development of infinite combined traces, as well as recognisable languages of combined traces.
\end{abstract}

\section{INTRODUCTION}

The dynamic behaviours of concurrent systems are usually described as sequences of atomic actions of such systems, which leads to its formal language semantics. Using this simple approach we cannot express some phenomena, e.g, concurrency and causality, that are crucial in the process of understanding and analysing concurrent behaviours of a system. In the case of a particular operational model, one can consider extending the sequential description by adding some information about the relevant properties of behaviours. One can do it by considering sequences of steps of actions and by adding some causal dependencies between actions. A well known approach that helps to capture concurrency and causality of a system are traces [CF69, Maz77].

Consider, for example, the elementary net system with inhibitor arcs in Example 1.1( $(a)$. We have four actions, $a, b, c$ and $d$, which may be executed in the initial marking, and two actions, $e$ and $f$, which need a previous history of computation to be enabled. Let us focus on action $e$. To enable this action we need to execute actions $a$ and $c$. We can execute

$2012 A C M C C S$ : [Theory of computation]: Models of computation-Concurrency.

Key words and phrases: concurrency, causal structures, combined traces, Mazurkiewicz traces, Petri nets, elementary net systems.

* A short variant of this paper, without proofs, appeared in the CONCUR 2012 conference proceedings. 
them together or in any order. To capture the concurrent behaviour of this computation we need to identify two sequences of executions - ace and cae. Using step semantics, which is not necessary in this case, we add also step sequence $(a c)(e)$ as another possible execution. Traces are sufficient to deal with such behaviours.

The situation is more complex in the case of action $f$. Now we need three tokens in the pre-set of the considered action, hence actions $b, c$ and $d$ should be executed before the action $f$. Because of the presence of inhibitors, there is only one way to execute them sequentially, they should be executed in the order $d c b f$. Note that $b d c f$ or $b c d f$ are not correct sequences of execution. There are, however, other possibilities to execute the four actions in the step semantics. For instance all three actions may be executed simultaneously as a step containing $b, c$ and $d$. This gives $(b c d)(f)$ as our allowed sequence of steps. Other step sequences are $(d)(b c)(f)$ and $(c d)(b)(f)$. It is important that action $d$ has to be executed not later than action $c$, and action $c$ has to be executed not later than action $b$. In this case traces are still applicable, but they lose some important behavioural information.

Another case is depicted in Example 1.1(b). The upper part of the net is identical to the first case. Here, however, there is a single action $g$ that waits for tokens in all four middle places. In other words, whole tuple $(a, b, c, d)$ has to be executed before action $g$. It is easy to see that because of inhibitors there is no valid sequential execution of the four actions. After executing one of these actions, one of the remaining becomes disallowed. The only possible execution is the step sequence $(a b c d)(f)$. Those two situations cannot be precisely described by traces, we need a more complex notion that capture "not later than" relationship between actions. To address this issue one can use a natural generalisation of traces called combined traces (see [JK95]).

Example 1.1. Two elementary net systems with inhibitor arcs.

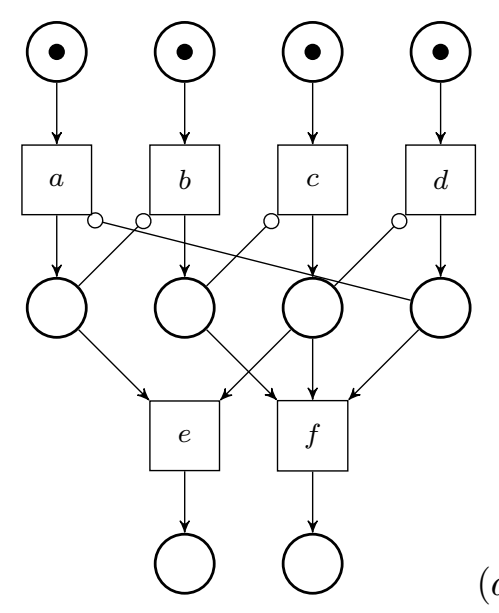

(a)

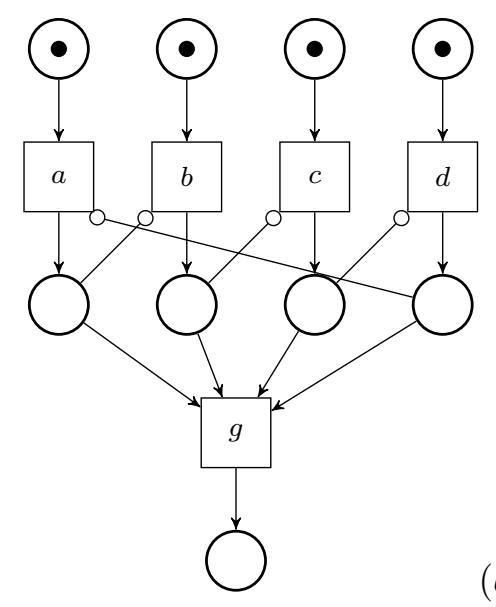

In this paper, we are concerned with the understanding of the algebraic inner structure of the combined traces (comtraces in short). We start by recalling some standard notions about formal languages, traces and comtraces. In particular, we give the definition of a lexicographical order on step sequences. We then recall the Foata canonical form of a comtrace that turns out to be maximal with respect to their order, and propose another canonical representative - the lexicographical canonical form. Then, we discuss the phenomenon of indivisibility in the case of comtraces and its connections with lexicographical canonical form. In the following sections, we propose an algebraic representation of a comtrace based 
on projections onto sequential subalphabets, and give a nondeterministic procedure that

allows to reconstruct step sequences of the original comtrace. We also give two strategies of determining such reconstruction, each leading to a proper canonical form of a comtrace. In the final section, we describe some natural applications of the algebraic properties developed in this paper, and sketch the directions for further research.

The preliminary version of this paper was presented on the CONCUR 2012 conference (Newcastle, UK) and published in local proceedings. The present paper is significantly extended and improved version.

\section{PReliminaries}

Throughout the paper we use the standard notions of the formal language theory. In particular, by an alphabet we mean a nonempty finite set $\Sigma$, the elements of which are called (atomic) actions. Finite sequences over $\Sigma$ are called words. The set of all finite words, including the empty word $\epsilon$, is denoted by $\Sigma^{*}$.

Let $w=a_{1} \ldots a_{n}$ and $v=b_{1} \ldots b_{m}$ be two words. Then

$$
w \circ v=w v=a_{1} \ldots a_{n} b_{1} \ldots b_{m}
$$

is the concatenation of $w$ and $v$. The alphabet $\operatorname{alph}(w)$ of $w$ is the set of all the actions occurring within $w$, and $\#_{a}(w)$ is the number of occurrences of an action $a$ within $w$. By $|w|$ we denote the length of word $w$. More generally, for an object $X$, whenever the notion of size is clear from the contexts, we denote its size by $|X|$.

Let $w=a_{1} \ldots a_{n}$ be a word. We use the notions of prefix and suffix of the word $w$. For any $k \leq n$, the $k$-suffix of $w$, denoted by $\operatorname{suff}_{k}(w)$, is a word $a_{k} \ldots a_{n}$. Similarly, the $k$-prefix of $w$, denoted by $\operatorname{pref}_{k}(w)$, is the word $a_{1} \ldots a_{k}$.

We assume that the alphabet $\Sigma$ is given together with a total order $\leq$, called lexicographical order and extend it to the level of words. Such an order is inherited from the first actions on which two words being compared differ. In the case that one word is a prefix of another - the former is the smaller one.

The projection onto a binary subalphabet $\{a, b\}$ is the function $\Pi_{a, b}: \Sigma^{*} \rightarrow \Sigma^{*}$ defined as follows:

$$
\Pi_{a, b}(c w)=\left\{\begin{array}{lll}
c \Pi_{a, b}(w) & \text { for } & c \in\{a, b\} \\
\Pi_{a, b}(w) & \text { for } & c \notin\{a, b\}
\end{array}\right.
$$

and $\Pi_{a, b}(\epsilon)=\epsilon$. In the same way we define a projection onto a unary subalphabet $\{a\}$, denoted by $\Pi_{a, a}: \Sigma^{*} \rightarrow \Sigma^{*}$.

The algebra of binary relations over set $X$ (i.e., subsets of $X \times X)$ is equipped with a concatenation operation $\circ$, where $R_{1} \circ R_{2}=\left\{(x, y) \mid \exists_{z \in X} x R_{1} y \wedge y R_{2} z\right\}$. The neutral element for $\circ$ is the identity relation $I_{X}=\{(x, x) \mid x \in X\}$, the index $X$ is omitted if it is clear from context. The $n$-th power of a relation $R$ is defined as $R^{n}=R^{n-1} \circ R$ for all $n \geq 1$, where $R^{0}=I$. The transitive closure of $R$ is $R^{+}=R^{1} \cup R^{2} \cup \ldots$, while its reflexive transitive closure is $R^{*}=R^{0} \cup R^{+}$. Moreover, for a relation $R \subset X \times X$ we define the reverse of $R$ by $R^{-1}=\{(x, y) \mid(y, x) \in R\}$, and its symmetric closure by $R^{\text {sym }}=R \cup R^{-1}$. We also define the largest equivalence relation contained in the reflexive and transitive closure of relation $R$ as

$$
R^{\circledast}=\left\{(x, y) \mid x R^{*} y \wedge y R^{*} x\right\} .
$$


The relation $R \subseteq X \times X$ is called symmetric if $R=R^{-1}$, reflexive if $I \subseteq R$, irreflexive if $I \cap R=\varnothing$, transitive if $R^{2} \subseteq R$, and acyclic if $R^{+}$is irreflexive. Moreover, for every $Y \subseteq X$ we define the restriction of the relation $R \subseteq X \times X$ to the set $Y$ by

$$
\left.R\right|_{Y}=\{(x, y) \in R \mid x, y \in Y\} .
$$

A directed acyclic graph is a pair $d a g=(X, R)$, where $X$ is a finite set and $R$ is an acyclic irreflexive binary relation on $X$. In a diagrammatical representation, $X$ is the set of vertices while $R$ the set of arcs. A directed acyclic graph $p o=(X, \prec)$ is a poset if the relation $\prec$ is transitive. An upper set is a nonempty subset $U$ of poset $p o=(X, \prec)$ such that for every $x \in U$ if $x \prec y$ then $y \in U$.

2.1. Elementary Net Systems with Inhibitor Arcs. In this paper we introduce some algebraic properties of combined traces which are the abstract model that describes causal relationships between executed actions of a concurrent system. The underlying structure, which was a motivation to define combined traces, are elementary net systems with inhibitor arcs.

Formally, the elementary net system with inhibitor arcs (or ENI - system) is a tuple $N=\left(P, T, F, I, M_{0}\right)$, where $P$ and $T$ are two disjoint and finite sets of places and transitions (or actions) respectively. Two other components, $F \subseteq(P \times T) \cup(T \times P)$ and $I \subseteq P \times T$ are relations, called flow relation and inhibition relation. These relations describe possible dynamic behaviours of a net, which are manifested by executing sets of enabled transitions called steps. Such an execution leads from one set of places (called marking) to another. The initial marking $M_{0} \subseteq P$, from which the action of a system begins, is the last element of the tuple $N$.

Given an ENI-system $N=\left(P, T, F, I, M_{0}\right)$ and $x \in P \cup T$, the pre-set (set of inputs) of $x$, denoted by $\bullet^{\bullet}$, is defined as $\bullet^{\bullet}=\{y \mid(y, x) \in F\}$, while the post-set (set of outputs) of $x$, denoted by $x^{\bullet}$, is defined as $x^{\bullet}=\{y \mid(x, y) \in F\}$. We also use the notion $\bullet x^{\bullet}$ for the union of the post-set and pre-set of $x$, calling it the set of neighbouring places/transitions (or simply the neighbourhood). Moreover, if $x \in T$, the inh-set (set of inhibitors) of $x$, denoted by ${ }^{\circ} x$, is defined by ${ }^{\circ} x=\{y \mid(y, x) \in I\}$. The set of neighbouring places together with the inh-set forms an extended neighbourhood of an action.

The dot notations are lifted in the usual way to sets of elements. Hence, by ${ }^{\bullet} X$ we denote the set $\{y \mid(y, x) \in F \wedge x \in X\}, X^{\bullet}=\{y \mid(x, y) \in F \wedge x \in X\}$, and ${ }^{\circ} X=\{y \mid(y, x) \in$ $I \wedge x \in X\}$. Graphically, the places are drawn as circles, transitions as rectangles, elements of flow relation as arcs, and elements of inhibition relation as arcs with small circles as arrowheads. Marked places are depicted by drawing small dot called token inside.

We say that a step $S=\left\{t_{1}, t_{2}, \ldots, t_{n}\right\}$ is enabled in marking $M$ if $\bullet \subseteq M, S^{\bullet} \cap M=\varnothing$, ${ }^{\circ} S \cap M=\varnothing$ and $\bullet_{i} \cap \bullet t_{j}=\varnothing$ for any $i \neq j$. The execution of such a step $S$ leads from the marking $M$ to the new marking $M^{\prime}=(M \backslash \bullet S) \cup S^{\bullet}$.

An ENI-system with empty inhibition relation, often considered under the sequential rather than step semantics, is called an elementary net system (or EN-system). 
Example 2.1. Consider a system $N=\left(P, T, F, I, M_{0}\right)$ depicted below.



The set of places has eight elements (from $p_{1}$ to $p_{8}$ ), the set of transitions has four elements $(T=\{a, b, c, d\})$. In the initial marking, three places are marked $-\left(p_{1}, p_{6}, p_{8}\right)$. Therefore, seven steps - including $(a),(d)$ and $(a d)$ - are enabled. Note that after executing transition $d$, transition a remains enabled, however, this does not hold in the opposite direction, i.e. after executing transition $a$ there is a token in place $p_{3}$ and transition $d$ is no more enabled.

2.2. Traces. In this section we recall well-known notion of traces (see DR95, Maz77, Mik08). Traces are an abstract model describing causal relationships between executed actions in, for example EN-systems. They capture independence, hence the possibility to be executed in any order (and also together) for some actions. Structurally, pairs of actions with disjoint sets of neighbouring places are in the independence relation.

A concurrent alphabet is a pair $\Psi=(\Sigma$, ind $)$, where $\Sigma$ is an alphabet and ind $\subseteq \Sigma \times \Sigma$ is an irreflexive and symmetric independence relation. The corresponding dependence relation is given by $d e p=(\Sigma \times \Sigma) \backslash i n d$.

A concurrent alphabet $\Psi$ defines an equivalence relation $\equiv_{\Psi}^{\Sigma}$ identifying words which differ only by the ordering of independent actions. Two words, $w, v \in \Sigma^{*}$, satisfy $w \equiv \sum_{\Psi}^{\Sigma} v$ if there exists a finite sequence of commutations of adjacent independent actions transforming $w$ into $v$. More precisely, $\equiv_{\Psi}^{\Sigma}$ is a binary relation over $\Sigma^{*}$ which is the reflexive and transitive closure of the relation $\sim_{\Psi}^{\Sigma}$ such that $w \sim_{\Psi}^{\Sigma} v$ if there are $u, z \in \Sigma^{*}$ and $(a, b) \in$ ind satisfying $w=u a b z$ and $v=u b a z$.

Equivalence classes of $\equiv_{\Psi}^{\Sigma}$ are called (Mazurkiewicz) traces and the trace containing a given word $w$ is denoted by $[w]$. The set of all traces over $\Psi$ is denoted by $\Sigma^{*} / \equiv_{\Psi}^{\Sigma}$, and the pair $\left(\Sigma^{*} / \equiv_{\Psi}^{\Sigma}, \circ\right)$ is a (trace) monoid, where $\tau \circ \tau^{\prime}=\left[w \circ w^{\prime}\right]$, for any words $w \in \tau$ and $w^{\prime} \in \tau^{\prime}$, is the concatenation operation for traces. Note that trace concatenation is well-defined as $\left[w \circ w^{\prime}\right]=\left[v \circ v^{\prime}\right]$, for all $w, v \in \tau$ and $w^{\prime}, v^{\prime} \in \tau^{\prime}$. Similarly, for every trace $\tau=[w]$ and every action $a \in \Sigma$, we can define

$$
\operatorname{alph}(\tau)=\operatorname{alph}(w) \quad \#_{a}(\tau)=\#_{a}(w) .
$$

Projections onto unary and binary dependent subalphabets (i.e. $\{a, b\} \subseteq \Sigma$ such that $(a, b) \in d e p)$ are invariants for traces (see [Mik08]). It is possible to formulate the trace equivalence in terms of projections. Two words $u, w \in \Sigma^{*}$ are in relation $\equiv_{\Psi}^{\Sigma}$ if and only if

$$
\forall(a, b) \in \operatorname{dep} \Pi_{a, b}(u)=\Pi_{a, b}(w) .
$$

Following [Mik08], we define the projection representation of $\tau$ as a function $\Pi_{\tau}: \operatorname{dep} \rightarrow \Sigma^{*}$, where $\Pi_{\tau}(a, b)=\Pi_{a, b}(\tau)$. 
Example 2.2. Consider a concurrent alphabet $\Psi$ with four actions $\Sigma=\{a, b, c, d\}$ together with a dependence relation $d e p$ given by:

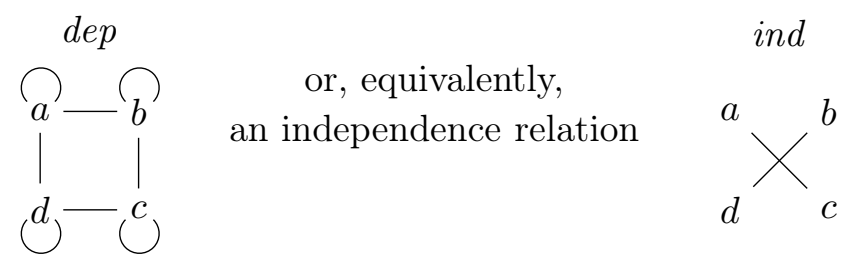

Then $w=$ abbaacd $\equiv_{\Psi}^{\Sigma}$ abbcaad.

The projection representation of a trace $\tau=[w]$ is

$$
\begin{array}{llll}
\Pi_{a, a}(\tau)=a a a & \Pi_{b, b}(\tau)=b b & \Pi_{c, c}(\tau)=c & \Pi_{d, d}(\tau)=d \\
\Pi_{a, b}(\tau)=a b b a a & \Pi_{a, d}(\tau)=a a a d & \Pi_{b, c}(\tau)=b b c & \Pi_{c, d}(\tau)=c d
\end{array}
$$

A word $w \in \Sigma^{*}$ is in Foata canonical form (see [DM97]) w.r.t. the dependence relation dep and a lexicographical order $\leq$ on $\Sigma$, if $w=w_{1} \ldots w_{n}(n \geq 0)$, where each $w_{i}$ is a nonempty word such that:

- $\operatorname{alph}\left(w_{i}\right)$ is pairwise independent and $w_{i}$ minimal w.r.t. lexicographical order $\leq$ among $\left[w_{i}\right]$

- for each $i>1$ and action $a$ occurring in $w_{i}$, there exists action $b$ occurring in $w_{i-1}$ such that $(a, b) \in$ dep.

Another canonical (normal) form of a trace that one may consider is the lexicographical canonical form (see also [DM97]). It is based only on the lexicographical order and is defined as the least representative of a trace with respect to the lexicographical ordering. The intuition behind the Foata canonical form is that it groups actions into maximally concurrent steps, while the lexicographical canonical form is very useful in some combinatorial approaches (see [MPS11]). Each trace contains exactly one sequence in the Foata canonical form, and exactly one sequence in the lexicographical canonical form. It may happen that the two versions of canonical form coincide.

2.3. Step Traces. Let us lift the notion of traces from the sequential semantics discussed above to the step semantics. Instead of identifying sequences of actions over alphabet $\Sigma$, we will identify sequences of sets of actions, called steps. We demand that a step should consist of mutually independent actions only.

For a given concurrent alphabet $\Psi=(\Sigma$, ind $)$ we define a set $\mathbb{S}_{\Psi}$ of all nonempty subsets $A \subseteq \Sigma$ such that for all $a, b \in A$ we have $a \neq b \Rightarrow(a, b) \in$ ind. If the concurrent alphabet $\Psi$ is clear from the context, we would write $\mathbb{S}$ instead of $\mathbb{S}_{\Psi}$. To avoid confusion with the wellestablished operation of concatenating sets in formal languages theory, we follow Diekert ([DM97]) and denote a step containing actions $a$ and $b$ by $(a b)$ rather then $\{a, b\}$, etc. Finite sequences in $\mathbb{S}^{*}$, including the empty one $\lambda=(\epsilon)$, are called step sequences.

We now lift a number of notions and notations introduced for words to the level of step sequences. In what follows, $\Psi=(\Sigma$, ind $)$ is a fixed concurrent alphabet. Let $w=A_{1} \ldots A_{n}$ and $v=B_{1} \ldots B_{m}$ be two step sequences. Then $w \circ v=w v=A_{1} \ldots A_{n} B_{1} \ldots B_{m}$ is the concatenation of $w$ and $v$. The alphabet $\operatorname{alph}(w)$ of $w$ comprises all actions occurring 
within $w$, and $\#_{a}(w)$ is the number of occurrences of an action $a$ within $w$. Moreover, we define the step alphabet $\operatorname{Alph}(w) \subseteq \mathbb{S}$ of a step sequence $w$ as the set of all steps occurring in $w$.

Both independence and dependence relations may be extended to the case of steps. Two steps $A, B \in \mathbb{S}$ are independent if and only if $A \times B \subseteq$ ind, otherwise they are dependent. We not only allow to commute, but also to join/split pairs of independent steps. In fact, the commutation of two independent steps may be composed as two join/split operations. More precisely, $\equiv_{\Psi}^{\mathbb{S}}$ is a binary relation over $\mathbb{S}^{*}$ which is the reflexive, symmetric and transitive closure of the relation $\sim_{\Psi}^{\mathbb{S}}$ such that $w \sim_{\Psi}^{\mathbb{S}} v$ if there are $u, z \in \mathbb{S}^{*}$ and $A, B \in \mathbb{S}$ satisfying $w=u A B z, v=u(A \cup B) z$, and $A \times B \subseteq$ ind. Note that $A \cap B=\varnothing$, since ind is irreflexive. Equivalence classes of $\equiv_{\Psi}^{\mathbb{S}}$ are called step traces (see Vog91). The trace containing a step sequence $w$ is denoted by $[w]$, while set of all step traces - by $\mathbb{S}^{*} / \equiv_{\Psi}^{\mathbb{S}}$. Step traces are a conservative extension of sequential traces. To justify this statement we prove

Proposition 2.3. Let $\Psi=(\Sigma$, ind $)$ be a concurrent alphabet, and $w, v$ two step sequences over $\Psi$. If $w=u A B z$ and $v=u B A z$, where $u, z \in \mathbb{S}^{*}$ and $A \times B \subseteq$ ind then $w \equiv_{\Psi}^{\mathbb{S}} v$.

Proof. Directly from the definition, both $w$ and $v$ are in the relation $\sim_{\Psi}^{\mathbb{S}}$ with $y=u(A \cup B) z$. Since $\equiv_{\Psi}^{\mathbb{S}}$ is the reflexive, symmetric and transitive closure of $\sim_{\Psi}^{\mathbb{S}}$, we have $w \equiv_{\Psi}^{\mathbb{S}} y$ and $v \equiv \underset{\Psi}{\mathbb{S}} y$, so also $w \equiv_{\Psi}^{\mathbb{S}} v$.

We define two operations which help to move from step semantics into sequential semantics and vice versa. Let $A \in \mathbb{S}$ be a step and $\leq$ be a total order on $\Sigma$. Using the relation $\leq$ we define $\min (A)$, the minimal representative of a step $\varnothing \neq A \in \mathbb{S}$ as the minimal action in $A$ with respect to $\leq$. Note that $\min (\varnothing)$ is not defined. We define the lexicographical linearization of step $A$ as

$$
\operatorname{lex}(A)=\left\{\begin{array}{lll}
\epsilon & \text { for } \quad A=\varnothing \\
\min (A) \operatorname{lex}(A \backslash \min (A)) & \text { for } \quad A \neq \varnothing .
\end{array}\right.
$$

We extend the operation lex to step sequences and sets of step sequences in the usual way:

$$
\begin{aligned}
& \operatorname{lex}\left(A_{1} A_{2} \ldots A_{n}\right)=\operatorname{lex}\left(A_{1}\right) \operatorname{lex}\left(A_{2}\right) \ldots \operatorname{lex}\left(A_{n}\right), \\
& \operatorname{lex}(X)=\{\operatorname{lex}(w) \mid w \in X\} .
\end{aligned}
$$

As a reverse operation, we define a singletonization of an action $a$ by $\operatorname{sstep}(a)=\{a\}$ and extend it to the case of sequences by $\operatorname{sstep}\left(a_{1} \ldots a_{n}\right)=\operatorname{sstep}\left(a_{1}\right) \ldots \operatorname{sstep}\left(a_{n}\right)$.

Since no two dependent actions may occur in the same step, we can easily lift the notion of projections onto unary and binary dependent subalphabets to the case of step sequences being representatives of step traces:

$$
\Pi_{a, b}(A w)=\left\{\begin{array}{lll}
a \Pi_{a, b}(w) & \text { for } & a \in A \\
b \Pi_{a, b}(w) & \text { for } & b \in A \\
\Pi_{a, b}(w) & \text { for } & \{a, b\} \cap A=\varnothing
\end{array}\right.
$$

and $\Pi_{a, b}(\lambda)=\epsilon$. Note that both actions $a$ and $b$ can not simultaneously be in $A$, since they are dependent.

Proposition 2.4. Let $\Psi=(\Sigma$, ind $)$ be a concurrent alphabet, and $w, u \in \mathbb{S}^{*}$ two step sequences over $\Psi$. Then $w \equiv_{\Psi}^{\mathbb{S}} u$ if and only if lex $(w) \equiv_{\Psi}^{\Sigma} \operatorname{lex}(u)$. 
Proof. Since every action in sequential semantics can be treated as a singleton step in step semantics, the implication $\operatorname{lex}(w) \equiv_{\Psi}^{\Sigma} \operatorname{lex}(u) \Rightarrow w \equiv_{\Psi}^{\mathbb{S}} u$ follows directly from Proposition 2.3. Therefore we need to prove that $w \equiv_{\Psi}^{\mathbb{S}} u \Rightarrow \operatorname{lex}(w) \equiv_{\Psi}^{\Sigma} \operatorname{lex}(u)$.

Recalling the definition of step traces, it is sufficient to show that for $A, B \in \mathbb{S}$ such that $A \times B \subseteq$ ind we have $\operatorname{lex}(A) \operatorname{lex}(B) \equiv_{\Psi}^{\Sigma} \operatorname{lex}(A \cup B)$. We make use of the projection formulation for sequential trace equivalence. Since $A \times B \subseteq$ ind we have $A \cap B=\varnothing$. Moreover $A \cup B$ is a step, hence for every dependent pair $(a, b)$ we have $\Pi_{a, b}(A \cup B)$ empty or equal to a single action (also in the degenerated case $a=b$ ).

Therefore for every $(a, b) \in$ dep we have either

$$
\Pi_{a, b}(A \cup B)=\Pi_{a, b}(A) \text { when }\{a, b\} \cap A \neq \varnothing \wedge\{a, b\} \cap B=\varnothing,
$$

or

$$
\Pi_{a, b}(A \cup B)=\Pi_{a, b}(B) \text { when }\{a, b\} \cap B \neq \varnothing \wedge\{a, b\} \cap A=\varnothing .
$$

Hence $\Pi_{a, b}(A \cup B)=\Pi_{a, b}(A) \Pi_{a, b}(B)$ and $\operatorname{lex}(A) \operatorname{lex}(B) \equiv{ }_{\Psi}^{\Sigma} \operatorname{lex}(A \cup B)$.

Proposition 2.5. Let $w$ be a step sequence over a concurrent alphabet $\Psi$. Then the sequences of singletons are fixpoints of the function sstep $\circ$ lex, i.e.

$$
\text { sstep } \circ \operatorname{lex}(w)=w \Longleftrightarrow \forall_{A \in A l p h(w)}|A|=1 \text {. }
$$

Proof.

$\Longrightarrow$ :

Let $w \in \mathbb{S}^{*}$ and $A \in \operatorname{Alph}(w)$ be such that $|A|>1$. Without loss of generality we may assume that $A$ is the first step in $w$. Then

$$
\operatorname{lex}(w)=\operatorname{lex}\left(A w^{\prime}\right)=\operatorname{lex}(A) \operatorname{lex}\left(w^{\prime}\right)=\min (A) \operatorname{lex}(A \backslash \min (A)) \operatorname{lex}\left(w^{\prime}\right),
$$

hence

$$
\text { sstep } \circ \text { lex }(w)=\operatorname{sstep}(\min (A)) \operatorname{sstep} \circ \operatorname{lex}\left((A \backslash \min (A)) w^{\prime}\right) .
$$

As a result we get that the first step in $\operatorname{sstep} \circ \operatorname{lex}(w)$ is a singleton, which is in contradiction with the assumption $|A|>1$. Hence $\forall_{A \in A l p h(w)}|A|=1$.

$\Longleftarrow$ :

The second implication is straightforward, since $|A|=1$ implies $A=\{a\}$ and $a=\min (A)$, so lex $(A)=a$ and sstep $\circ \operatorname{lex}(A)=\operatorname{sstep}(a)=\{a\}=A$. Let $w=A_{1} \ldots A_{n}$. Directly from the definitions

$$
\text { sstep } \circ \text { lex }(w)=\text { sstep } \circ \text { lex }\left(A_{1}\right) \ldots \text { sstep } \circ \operatorname{lex}\left(A_{n}\right)=A_{1} \ldots A_{n}=w .
$$

Proposition 2.6. Let $u$ be a sequence over a concurrent alphabet $\Psi$. Then lexosstep $(u)=u$.

Proof. Let $u=a_{1} \ldots a_{n}$ and $A_{i}=\left\{a_{i}\right\}$. Analogously to the proof of Proposition 2.5, we have $a_{i}=\operatorname{lex}\left(A_{i}\right)$ and $\operatorname{sstep}\left(a_{i}\right)=A_{i}$, so

$$
\begin{aligned}
\operatorname{lex} \circ \operatorname{sstep}(u)=\operatorname{lex} \circ \operatorname{sstep}\left(a_{1} \ldots a_{n}\right)=\operatorname{lex}\left(\operatorname{sstep}\left(a_{1}\right) \ldots \operatorname{sstep}\left(a_{n}\right)\right) \\
\quad=\operatorname{lex}\left(A_{1} \ldots A_{n}\right)=\operatorname{lex}\left(A_{1}\right) \ldots \operatorname{lex}\left(A_{n}\right)=a_{1} \ldots a_{n}=u .
\end{aligned}
$$


The pair $\left(\mathbb{S}^{*} /_{\equiv_{\Psi}^{\mathbb{S}}}, \circ\right)$ is a (step trace) monoid, where $\tau \circ \tau^{\prime}=\left[w \circ w^{\prime}\right]$, for any step sequences $w \in \tau$ and $w^{\prime} \in \tau^{\prime}$. Step trace concatenation is well-defined as $\left[w \circ w^{\prime}\right]=\left[v \circ v^{\prime}\right]$, for all $w, v \in \tau$ and $w^{\prime}, v^{\prime} \in \tau^{\prime}$. A step trace $\tau$ is a prefix of a step trace $\tau^{\prime}$ if there is a step trace $\tau^{\prime \prime}$ such that $\tau \circ \tau^{\prime \prime}=\tau^{\prime}$. As in the case of sequential traces, for every step trace $\tau$ and every $a \in \Sigma$, we can define $\operatorname{alph}(\tau)=\operatorname{alph}(w)$ and $\#_{a}(\tau)=\#_{a}(w)$, where $w$ is any step sequence belonging to $\tau$. The situation with the step alphabet, as it is not an invariant of a step trace, is a bit more complex: we define $\operatorname{Alph}(\tau)=\bigcup_{w \in \tau} \operatorname{Alph}(w)$.

Theorem 2.7. Let $\Psi$ be a concurrent alphabet, and $w, u \in \mathbb{S}^{*}$. Then $w \equiv_{\Psi}^{\mathbb{S}} u$ if and only if $\forall(a, b) \in d e p=\Pi_{a, b}(w)=\Pi_{a, b}(u)$.

Proof. The proof follows directly from Proposition 2.4 and the projection based definition of trace equivalence (in sequential semantics).

Next, we give the canonical (normal) form of a step trace which essentially captures a greedy, maximally concurrent, execution of the actions occurring in the step trace conforming to the independence relations. A step sequence $w=A_{1} \ldots A_{n} \in \mathbb{S}^{*}$ is in Foata canonical form if, for each $i \leq n$, whenever $A v \equiv_{\Psi}^{\mathbb{S}} A_{i} \ldots A_{n}$ for some $A \in \mathbb{S}$ and $v \in \mathbb{S}^{*}$, then $A \subseteq A_{i}$. One can see that all suffixes and all prefixes of a step sequence in Foata canonical form are also in Foata canonical form, and that each step trace comprises a unique step sequence in Foata canonical form. Note that the following statement holds:

Proposition 2.8. Let $\Psi$ be a concurrent alphabet. A step sequence $w=A_{1} \ldots A_{n} \in \mathbb{S}^{*}$ is in Foata canonical form if and only if for every $i<n$, there is no $\varnothing \neq A \subseteq A_{i+1}$ such that $A_{i} \times A \subseteq$ ind.

Proof.

$\Longrightarrow$ :

Let $w=A_{1} \ldots A_{n}$ be in Foata canonical form. Suppose that there are $i<n$ and $\varnothing \neq A \subseteq A_{i}$ such that $A \times A_{i} \subseteq$ ind. Then, since for every $A_{k}$ we have $A_{k} \times A_{k} \subseteq($ ind $\cup I)$,

$$
A_{i} A_{i+1} \ldots A_{n} \equiv{ }_{\Psi}^{\mathbb{S}} A_{i} A\left(A_{i+1} \backslash A\right) A_{i+2} \ldots A_{n} \equiv_{\Psi}^{\mathbb{S}}\left(A_{i} \cup A\right)\left(A_{i+1} \backslash A\right) A_{i+2} \ldots A_{n},
$$

and by $A \neq \varnothing$ we obtain that $w$ is not in Foata canonical form. Hence there are no such $i<n$ and $A \subseteq A_{i+1}$.

$\Longleftarrow$ :

Let $w=A_{1} \ldots A_{n}$ and for every $i<n$, there is no $\varnothing \neq A \subseteq A_{i+1}$ such that $A_{i} \times A \subseteq$ ind. Assume moreover that $A v \equiv_{\Psi}^{\mathbb{S}} A_{i} \ldots A_{n}$ and let $B=A_{i} \backslash A$. Suppose that $B$ is not empty and let $j$ be the least index such that $B \cap A_{j} \neq \varnothing$. By Theorem 2.7 not only such $j$ exist, but also we get that $B \times A_{k} \subseteq$ ind for every $i<k<j$. Hence $B \cap A_{j}$ is a nonempty step contained in $A_{j}$ and $\left(B \cap A_{j}\right) \times A_{j-1} \subseteq$ ind, which gives a contradiction with the assumptions, and so $B$ has to be empty, which ends the proof.

We can also distinguish one of the representatives of $\tau$, built from singletons. Note that such step sequences may be considered as sequences over $\Sigma$ and compared using lexicographical order $\leq$. Similarly to the case of sequential traces, we call the least (with respect to the order $\leq$ ) singleton based representative of a step trace $\tau$ its lexicographical canonical form.

Canonical forms of sequential and step traces connect those two worlds. More precisely, the following hold: 
Theorem 2.9. Let $\Psi=(\Sigma$, ind $)$ be a concurrent alphabet. Then a step sequence $w$ is in lexicographical canonical form if and only if all $A \in A l p h(w)$ are singletons and the sequence lex $(w)$ is in lexicographical canonical form.

Proof. According to Proposition 2.5, it is only an equivalent reformulation of the definition.

Theorem 2.10. Let $\Psi=(\Sigma$, ind $)$ be a concurrent alphabet. If a step sequence $w=$ $A_{1} A_{2} \ldots A_{k}$ is in Foata canonical form then the sequence $u=l e x(w)$ is in Foata canonical form.

Proof. We have to prove that sequences $l e x\left(A_{1}\right), \ldots, \operatorname{lex}\left(A_{k}\right)$ satisfy the conditions from the definition of Foata canonical form in the case of sequential trace. The elements of every $A_{i}$ are pairwise independent since $A_{i}$ is a step. Let us suppose that there exist $1<i \leq k$ and $a \in A_{i}$ such that $a$ is independent with every action $b$ from $A_{i-1}$. Let $A=\{a\}$. Then $A \neq \varnothing, A \subseteq A_{i}$, and $A_{i-1} \times A \subseteq i n d$. From the definition of Foata canonical form in the case of step traces we have that $w$ is not in Foata canonical form. Hence, $u$ is indeed in Foata canonical form.

Theorem 2.11. Let $\Psi=(\Sigma$, ind $)$ be a concurrent alphabet. If a sequence $u=a_{1} a_{2} \ldots a_{n}$ is in Foata canonical form then there exists a step sequence $w=A_{1} A_{2} \ldots A_{k}$ in Foata canonical form such that $u=l e x(w)$.

Proof. From the definition of Foata canonical form, we know that there exist sequences $u_{1}, \ldots, u_{k}$ such that the elements of $\operatorname{alph}\left(u_{i}\right)$ are pairwise independent for every $0<i \leq k$. Hence, for every $0<i \leq k$ we have that $\operatorname{alph}\left(w_{i}\right)$ is a step over $\Psi$. Let $A_{i}=\operatorname{alph}\left(w_{i}\right)$. Suppose that $w$ is not in Foata canonical form. Then, there exist nonempty $A \in \mathbb{S}$ and $0<i<k$ such that $A \subseteq A_{i+1}$ and $A_{i} \times A \subseteq$ ind. Let $a \in A$. Since $A_{i} \times A \subseteq$ ind there is no $b \in A$ dependent with $a$. Hence $u$ is not in Foata canonical form. This contradicts the assumptions and proves the theorem.

We conclude this subsection by formulating and proving a result that establishes a relationship between two semantics in which we can consider traces:

Theorem 2.12. Let $\sigma \in \Sigma^{*} / \equiv_{\Psi}^{\Sigma}$ be a trace (in sequential semantics). Then there exists a unique step trace $\tau$ such that lex $(\tau)=\sigma$.

Proof. Theorems 2.10 and 2.11 allow us to associate sequential trace $\sigma$ with a trace $\tau$ using their Foata canonical forms.

Let $w$ be a lexicographical canonical form of $\tau$. By Theorems 2.9 and 2.7, and definition of sequential traces based on projections, we get that $l e x(w) \in \sigma$ and lexicographical canonical forms of $\sigma$ and $\tau$ also overlaps. By Proposition 2.4 we conclude that $\operatorname{lex}(\tau) \subseteq \sigma$.

Let $X=\{\operatorname{sstep}(u) \mid u \in \sigma\}$. By Propositions 2.6] and 2.4 we get that $X \subseteq \tau$ and $\operatorname{lex}(X)=\sigma$. Hence $\sigma=\operatorname{lex}(X) \subseteq \operatorname{lex}(\tau)$ which ends the proof.

2.4. Comtraces. Whereas traces are satisfactory to describe the concurrent behaviour of EN-systems, they are not sufficient to capture the behaviour of systems with inhibitor arcs. To deal with such systems, we recall the notion of combined traces (see [JK95]).

A comtrace alphabet is a triple $\Theta=(\Sigma$, sim, ser $)$, where $\Sigma$ is an arbitrary alphabet and ser $\subseteq$ sim $\subseteq \Sigma \times \Sigma$ are two relations, respectively called serialisability and simultaneity; it 
is assumed that sim is irreflexive and symmetric. Intuitively, if $(a, b) \in \operatorname{sim}$ then $a$ and $b$ may occur simultaneously, whereas $(a, b) \in$ ser means that in such a case $a$ may also occur before $b$ (with both executions being equivalent). The set of all (potential) steps over $\Theta$, or step alphabet, is then defined as the set $\mathbb{S}_{\Theta}$ comprising all nonempty sets of actions $A \subseteq \Sigma$ such that $(a, b) \in \operatorname{sim}$, for all distinct $a, b \in A$. If the comtrace alphabet $\Theta$ is clear from the context, we would write $\mathbb{S}$ instead of $\mathbb{S}_{\Theta}$.

The comtrace congruence over $\Theta$, denoted by $\equiv_{\Theta}$, is the reflexive, symmetric and transitive closure of the relation $\sim_{\Theta} \subseteq \mathbb{S}^{*} \times \mathbb{S}^{*}$ such that $w \sim_{\Theta} v$ if there are $u, z \in \mathbb{S}^{*}$ and $A, B \in \mathbb{S}$ satisfying $w=u A B z, v=u(A \cup B) z$ and $A \times B \subseteq$ ser. Note that $A \cap B=\varnothing$ as ser is irreflexive.

Equivalence classes of the relation $\equiv_{\Theta}$ are called comtraces (see [JKK11]), and the comtrace containing a given step sequence $w$ is denoted by $[w]$. The set of all comtraces is denoted by $\mathbb{S}^{*} / \equiv_{\Theta}$, and the pair $\left(\mathbb{S}^{*} / \equiv_{\Theta}, \circ\right)$ is a (comtrace) monoid, where $\tau \circ \tau^{\prime}=\left[w \circ w^{\prime}\right]$, for any step sequences $w \in \tau$ and $w^{\prime} \in \tau^{\prime}$. Comtrace concatenation is well-defined as $\left[w \circ w^{\prime}\right]=\left[v \circ v^{\prime}\right]$, for all $w, v \in \tau$ and $w^{\prime}, v^{\prime} \in \tau^{\prime}$. A comtrace $\tau$ is a prefix of a comtrace $\tau^{\prime}$ if there is a comtrace $\tau^{\prime \prime}$ such that $\tau \circ \tau^{\prime \prime}=\tau^{\prime}$. As in the case of step traces, for every comtrace $\tau$ and every $a \in \Sigma$, we can define $\operatorname{alph}(\tau)=\operatorname{alph}(w)$ and $\#_{a}(\tau)=\#_{a}(w)$, where $w$ is any step sequence belonging to $\tau$. Moreover, $\operatorname{Alph}(\tau)=\bigcup_{w \in \tau} \operatorname{Alph}(w)$.

Next, we give the canonical form of a comtrace which essentially captures a greedy, maximally concurrent, execution of the actions occurring in the comtrace conforming to the simultaneity and serialisability relations. A step sequence $w=A_{1} \ldots A_{n} \in \mathbb{S}^{*}$ is in Foata canonical form if, for each $i \leq n$, whenever $A v \equiv_{\Theta} A_{i} \ldots A_{k}$ for some $A \in \mathbb{S}$ and $v \in \mathbb{S}^{*}$, then $A \subseteq A_{i}$. This canonical form of a comtrace is extensively discussed in [JL11]. One can see that all suffixes and all prefixes of step sequence in Foata canonical form are also in Foata canonical form, and that each comtrace comprises a unique step sequence in Foata canonical form.

Note that an alternative (equivalent) definition of normal form requires that, for every $i<k$, there is no $\varnothing \neq A \subseteq A_{i+1}$ such that $A_{i} \times A \subseteq$ ser and $A \times\left(A_{i+1} \backslash A\right) \subseteq$ ser. Moreover, in the cases of sequential and step traces we define two canonical forms. The first is, as in the case of comtraces, Foata canonical form, while the latter is called lexicographical. Both of those canonical forms prove to be very elegant and useful theoretical tool (as an example see prove of Theorem 2.12). In the next section we define the lexicographical canonical form of a comtrace. It is one of the main notions introduced and utilised in this paper. But previously, let us discuss in detail direct relationships between atomic actions.

2.5. Relations between actions. In our discussion, we use a number of relations capturing semantically meaningful relationships between individual actions (see also [MK11]):

- Dependence $d e p=(\Sigma \times \Sigma) \backslash \operatorname{sim}$, and independence ind $=\operatorname{ser} \cap \operatorname{ser}^{-1}$.

Both relations have their counterparts in trace theory, and we denote them in the same way. If two actions are dependent then they never occur in a common step. Two actions are independent if they can be executed in any order as well as simultaneously (as ser $\subseteq$ sim).

- Semi-independence $\sin =\operatorname{sim} \backslash \operatorname{ser}$.

In contrast to the situation found in traces, dependence and independence do not describe all possible relationships between individual actions in comtraces. The remaining ones are 
called, due to the possibility of occurring together without being fully independent, semiindependent actions. Semi-independent actions may be further divided into symmetric and antisymmetric parts:

- Strong simultaneity $\operatorname{ssm}=\operatorname{sim} \backslash\left(\operatorname{ser} \cup \operatorname{ser}{ }^{-1}\right)=\sin \backslash \operatorname{ser}^{-1}$.

If two actions are strongly simultaneous then may occur simultaneously but cannot be serialised at all. This means that two occurrences of strongly simultaneous actions which appear together in a step sequence $w$ would appear together in every step sequence belonging to the comtrace $[w]$.

- Weak dependence $w d p=\operatorname{ser}^{-1} \backslash \operatorname{ser}=\sin \backslash \sin ^{-1}$.

Two actions are weakly dependent if they can be serialised only in one way. This means that for any two actions $(a, b) \in w d p$, if their occurrences appear in the order ' $a$ followed by $b$ ' then they behave like completely dependent actions, while appearing in the order ' $b$ followed by $a$ ' allows one to equivalently execute (if there are no other obstacles) a step $(a b)$.

The main motivation to define all those classes was to capture the essence of the interplay between single atomic elements of concurrent systems modelled using comtraces. As a result we achieve the projection representation defined later.

Similarly to the case of simultaneity and serialisability, each of proposed relations can be described semantically by specific relationships between pre-sets, post-sets and inh-sets of pairs of actions. Note that if the set of neighbouring places of two actions overlaps, then those places are automatically considered as dependent (like in the case of traces and ENsystems). The main role in the further partition is played by the extended neighbourhoods. To capture dependence we have to add (to the overlapping of strict neighbourhoods) the situation when one action has an input place that is simultaneously an inhibitor for the other.

The intersections of post-sets and inh-sets of two different actions are significant if they are not dependent. Namely, if they are totally disjoint, which means that their extended neighbourhoods are disjoint, those two actions are independent. Remaining situations correspond to the cases when two action have disjoint neighbourhoods as well as disjoint pre-sets and inh-sets, but still overlapping extended neighbourhoods and are captured by the semiindependence relation.

Note that in the favourable circumstances both of them might be executable (like in the case of independence), but the execution of one of them may disable the execution of the other. If $\left({ }^{\circ} b \cap a^{\bullet}\right) \neq \varnothing \wedge\left({ }^{\circ} a \cap b^{\bullet}\right)=\varnothing$ then after executing $b$ we can immediately execute $a$, but not vice versa. While if both ${ }^{\circ} b \cap a^{\bullet}$ and ${ }^{\circ} a \cap b^{\bullet}$ are nonempty then we cannot split simultaneous execution of $a$ and $b$. The following table gives a straightforward description of all seven relations for ENI-systems. 


\begin{tabular}{|l|c|l|}
\hline $\begin{array}{l}\text { simultaneity } \\
\text { serialisability }\end{array}$ & $\begin{array}{l}(a, b) \in \operatorname{sim} \\
(a, b) \in \operatorname{ser}\end{array}$ & $\begin{array}{l}\bullet a^{\bullet} \cap \bullet^{\bullet}=\varnothing \wedge\left({ }^{\circ} a \cap{ }^{\bullet} b\right) \cup\left({ }^{\circ} b \cap{ }^{\bullet} a\right)=\varnothing \\
(a, b) \in \operatorname{sim} \wedge a^{\bullet} \cap\left(\bullet b \cup{ }^{\circ} b\right)=\varnothing\end{array}$ \\
\hline dependence & $(a, b) \in \operatorname{dep}$ & $\bullet a \bullet \cap \bullet b^{\bullet} \neq \varnothing \vee\left({ }^{\circ} a \cap \bullet^{\bullet} b\right) \cup\left({ }^{\circ} b \cap{ }^{\bullet} a\right) \neq \varnothing$ \\
independence & $(a, b) \in \operatorname{ind}$ & $(a, b) \notin \operatorname{dep} \wedge\left({ }^{\circ} a \cap b^{\bullet}\right) \cup\left({ }^{\circ} b \cap a^{\bullet}\right)=\varnothing$ \\
semi-independence & $(a, b) \in \sin$ & $(a, b) \notin \operatorname{dep} \wedge\left({ }^{\circ} b \cap a^{\bullet}\right) \neq \varnothing$ \\
strong simultaneity & $(a, b) \in \operatorname{ssm}$ & $(a, b) \notin \operatorname{dep} \wedge\left({ }^{\circ} b \cap a^{\bullet}\right) \neq \varnothing \wedge\left({ }^{\circ} a \cap b^{\bullet}\right) \neq \varnothing$ \\
weak dependence & $(a, b) \in w d p$ & $(a, b) \notin \operatorname{dep} \wedge\left({ }^{\circ} b \cap a^{\bullet}\right) \neq \varnothing \wedge\left({ }^{\circ} a \cap b^{\bullet}\right)=\varnothing$ \\
\hline
\end{tabular}

Example 2.13. Consider a comtrace alphabet $\Theta$ for ENI-system $N$ from Example 2.1. The simultaneity and serialisability relations are given by:

$$
\operatorname{sim}=\mid \begin{aligned}
& a-b \\
& d \sum c
\end{aligned} \quad \text { ser }=\uparrow_{\uparrow}^{a \rightleftharpoons b}
$$

In the net $N$ we have a pair of independent actions $(a, b)$. Note that their extended neighbourhoods are disjoint. The only pair of different and dependent actions is $(b, d)$. The reason for their dependency is the non-emptiness of their neighbourhoods. All the remaining pairs of different actions are semi-independent. Only one of them, namely $(a, c)$, is strongly simultaneous. Note that the post place of one of these actions is an inhibitor place of another, forming in the net graph a special kind of cycle. Similar behaviour (post place which is simultaneously inhibitor place), may be observed in the remaining cases, namely for pairs $(a, d),(d, c)$ and $(c, b)$. However, we have there an asymmetric situation and those pairs of actions are weakly dependent. The five derived relations on actions are as follows:

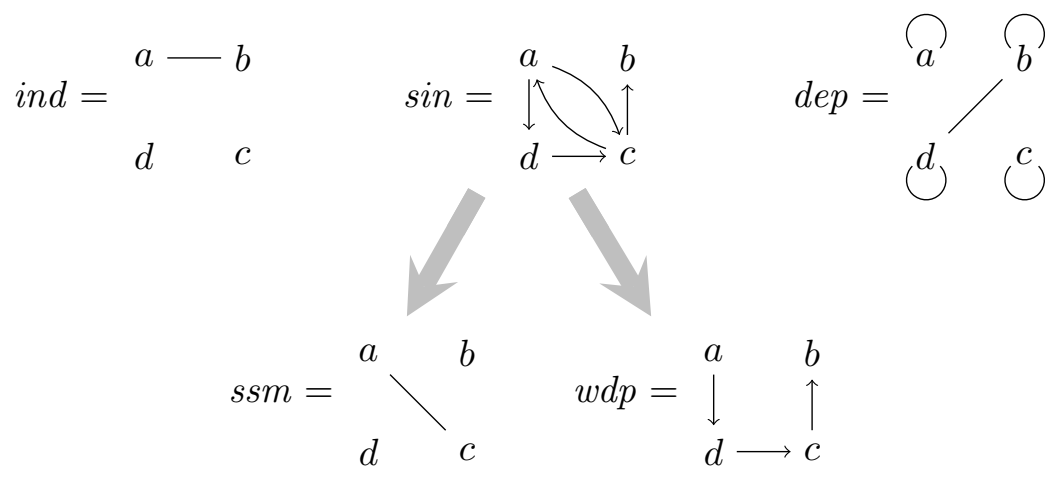

The combined trace of one of the possible executions in the net $N$ is $\tau=\{w, v, u, z\}$, where:

$$
\begin{aligned}
w & =(d)(a b) \\
v & =(d)(a)(b) \\
u & =(a d)(b) \\
z & =(d)(b)(a) .
\end{aligned}
$$

Moreover, $u$ is a step sequence in Foata canonical form. 


\section{LEXICOGRAPHICAL CANONICAL FORM}

We extend the order on actions to the case of steps (sets of actions). Let $A, B \in \mathbb{S}$ be two steps. If the size of $A$ is smaller then the size of $B$ then $A \widehat{\leq} B$. If the sizes are equal, $A \widehat{\leq} B$ if $A=B$ or $A \neq B$ and $\min (A \backslash B) \leq \min (B \backslash A)$. In this way, $(\mathbb{S}, \widehat{\leq})$ becomes a totally ordered set.

Using the order $\hat{\leq}$ we can define lexicographical order on step sequences in the usual way. The lexicographical canonical form of a comtrace $\tau$, denoted by minlex $(\tau)$, is the least (with respect to the lexicographical order $\widehat{\leq}$ ) step sequence contained in the comtrace. Note that, in contrast to the Foata canonical form, the lexicographical canonical form captures one of the most sequential executions of a comtrace. Hence the two canonical forms lie on the opposite sides of the concurrent/sequential spectrum of behaviours. Note that the step sequence $v$ from Example 2.13 is in lexicographical canonical form (assuming $a<b<c<d$ ).

Theorem 3.1. For a given comtrace $\tau$, its Foata canonical form is the $\hat{\leq}$-greatest, and its lexicographical canonical form is the $\widehat{\underline{\leq}}$-least, step sequence contained in $\bar{\tau}$.

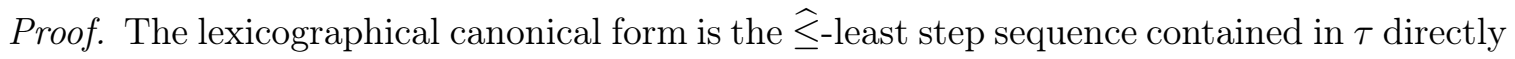
from the definition. We need to prove that Foata canonical form is greater than any other step sequence contained in $\tau$.

Let $u=A_{1} \ldots A_{n}, v=B_{1} \ldots B_{m}, u \neq v, u \equiv_{\Theta} v$, and $u$ be in Foata canonical form. Moreover, let $i=\min \left\{k \mid k \leq n \wedge A_{k} \neq B_{k}\right\}$. Note that such a number $i$ exists, since $u \neq v$ and $u \equiv_{\Theta} v$ so one sequence cannot be a prefix of another.

We have $A_{1} \ldots A_{k-1}=B_{1} \ldots B_{k-1}$, so directly form the definition of Foata canonical form $B_{k} \neq A_{k} \wedge B_{k} \subseteq A_{k}$. Since $B_{k} \widehat{\leq} A_{k}$, we have $B_{k} \ldots B_{m} \widehat{\leq} A_{k} \ldots A_{n}$, and $v \widehat{\leq} u$.

3.1. Indivisible steps and sequences. The structure and semantics of relations sim and ser mean that some actions have to appear simultaneously in every step sequence contained in a comtrace (in other word, they cannot be separated according to the comtrace congruence). A very good example of such actions are those in the ssm relation. The strong simultaneity, however, does not exhaust all situations when actions are "glued" together in a permanent manner. Such a behaviour was used in MK11 to form so called folded actions. It is also worth to observe that the notion of indivisible steps was discussed, in the case of step traces with auto-concurrency, in Vog91. In this section, we discuss the phenomenon of the indivisibility (in the case of comtraces) in depth.

Let us consider a step $A \in \mathbb{S}$ and a relation $\equiv_{A} \subseteq A \times A$, such that, for all $a, b \in A$, we have $a \equiv_{A} b$ if $(a, b) \in\left(\left.\sin \right|_{A}\right)^{\circledast}$. Intuitively, the relation $\equiv_{A}$ joins actions that can be executed simultaneously, but cannot be executed in a sequential way (see Example 3.2). Note that, for arbitrary step $A$, the relation $\equiv_{A}$ is an equivalence relation.

We say that a step $A \in \mathbb{S}$ is indivisible if $\forall_{a, b \in A} a \equiv_{A} b$. The set of all indivisible steps is denoted by $\widehat{\mathbb{S}}$. By $\operatorname{indiv}(\tau)$ we denote the set of all step sequences contained in a comtrace $\tau$ and built with indivisible steps only.

Example 3.2. Let us recall the comtrace alphabet from Example 2.13 and the relations sim and sin, which are crucial in determining indivisible steps. 


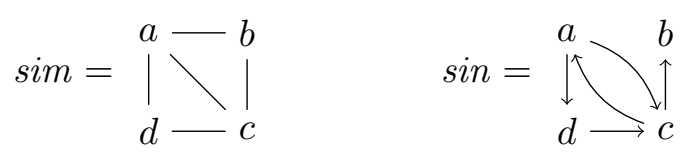

The set of all possible steps is $\mathbb{S}=\{(a),(b),(c),(d),(a b),(a c),(a d),(b c),(c d),(a b c),(a c d)\}$, while the set of all indivisible steps is $\widehat{\mathbb{S}}=\{(a),(b),(c),(d),(a c),(a c d)\}$. Note that step $A=(a b c)$ is divided by the relation $\equiv_{A}$ into two indivisible steps $B=(b)$ and $C=(a c)$ and step $B$ occurs not later than step $C$, while step $D=(a b)$ is divided by the relation $\equiv_{D}$ into two, completely independent, indivisible steps $(a)$ and $(b)$.

Moreover, there are only two sequences of indivisible steps contained in the comtrace $\tau$ which is defined in Example 2.13. These two sequences are $v=(d)(a)(b)$ and $z=(d)(b)(a)$.

Intuitively, we can treat the indivisible step sequences belonging to $\operatorname{indiv}(\tau)$ as classical sequences over the alphabet $\widehat{\mathbb{S}}$. Hence we define two complementary relations over this alphabet, the independence relation $\widehat{i n d}$ and the dependence relation $\widehat{d e p}$. We say that two indivisible steps $A$ and $B$ are independent if $A \times B \subseteq$ ind $=\operatorname{ser} \cap \operatorname{ser}^{-1}$; otherwise two indivisible steps are dependent.

Proposition 3.3. All steps contained in the lexicographical canonical form of a comtrace are indivisible $(\operatorname{minlex}(\tau) \in \operatorname{indiv}(\tau))$.

Proof. Suppose, to the contrary, that $\operatorname{minlex}(\tau)=u A v$ contains a non-indivisible step $A$. We conclude from Lemma 3.4 that for two disjoint steps $B$ and $C$ we have a step sequence $u B C v \in \tau$ which is different from the step sequence minlex $(\tau)$. Since $B \subseteq A$ and $A \neq B$ we have $u B C v \hat{\leq} u A v$ so we found a step sequence contained in $\tau$ that is lexicographically smaller than minlex $(\tau)$, which contradicts our assumption. Hence all steps contained in $\operatorname{minlex}(\tau)$ are indivisible.

Recall the lex operator defined in Section 2.3. It allows us to translate a step sequence to a sequence of actions, and was very helpful in dealing with step traces. In the case of comtraces, however, it has rather narrower application. Therefore, we define the split operator that translates arbitrary step sequences to step sequences of indivisible steps as $\widehat{\imath}: \mathbb{S}^{*} \rightarrow \widehat{\mathbb{S}}^{*}$ as

$$
\left(\widehat{A_{1} \ldots A_{n}}\right)=\widehat{A_{1}} \ldots \widehat{A_{n}}=\operatorname{minlex}\left(A_{1}\right) \ldots \operatorname{minlex}\left(A_{n}\right)
$$

The following facts justify an observation that the split operator does not lead beyond the comtrace, see Proposition 3.7

Lemma 3.4. Let $A \in \mathbb{S} \backslash \widehat{\mathbb{S}}$ be a step that is not indivisible. Then there exist two steps, $B$ and $C$, such that $A \sim_{\Theta} B C$. Moreover, $A / \equiv_{A}=B / \equiv_{B} \cup C / \equiv_{C}$.

Proof. Since $A$ is not indivisible, the relation $\equiv_{A}$ divides $A$ into at least two equivalence classes. In the following proof we choose an indivisible step, to play a role of $B$. However, at first we separate a special subset of $A$, denoted by $D$. One can think about $D$ as a set of elements from $A$, which form a minimal layer in the graph of the relation $\left(\left.\sin \right|_{A}\right)^{*}$.

Let $D$ be the set of all actions $b \in A$ such that, $\forall_{a \in A}(b, a) \in\left(\left.\sin \right|_{A}\right)^{*} \Rightarrow a \in[b]_{\equiv_{A}}$. Suppose that $D$ is empty. Let us take any $b_{1} \in A$. Then, by $D=\varnothing$, there exists $b_{2} \in A$ such that $b_{2} \notin\left[b_{1}\right]_{\equiv_{A}}$ and $\left(b_{1}, b_{2}\right) \in\left(\left.\sin \right|_{A}\right)^{*}$. Continuing in this way, we can construct an infinite sequence of actions $b_{i} \in A$ such that, for all $i, b_{i+1} \notin\left[b_{1}\right]_{\equiv_{A}} \wedge\left(b_{i}, b_{i+1}\right) \in\left(\left.\sin \right|_{A}\right)^{*}$. 
Since $A$ is finite, the elements contained in this sequence have to repeat. Let $b_{n}=b_{m}$ and $n<m$. Since $\left(\left.\sin \right|_{A}\right)^{*}$ is transitive we have $\left(b_{n+1}, b_{n}\right) \in\left(\left.\sin \right|_{A}\right)^{*}$ and $\left(b_{m}, b_{n+1}\right) \in\left(\left.\sin \right|_{A}\right)^{*}$, so $b_{n+1} \in\left[b_{n}\right]_{\equiv_{A}}$ which contradicts the assumption. Hence $D$ is not empty.

Let $d$ be an arbitrary element from $D$ and $B=[d]_{\equiv_{A}} . A$ is not indivisible, hence $A \neq B$. Moreover, directly from the construction of the set $D, B \subseteq D$. Let $b \in B$ and $a \in A \backslash B$. From the definition of $D$ we have that $(b, a) \notin\left(\left.\sin \right|_{A}\right)^{*}$, so $(b, a) \notin w d p$ and $(b, a) \notin s s m$. We also have $(a, b) \notin d e p$ since $a$ and $b$ are both contained in $A$. This gives

$$
\forall_{a \in A \backslash B} \forall_{b \in B}(a, b) \in w d p \vee(a, b) \in \text { ind. }
$$

Hence

and finally $A \sim_{\Theta} B(A \backslash B)$.

$$
\forall_{a \in A \backslash B} \forall_{b \in B}(b, a) \in \operatorname{ser}
$$

It remains to be proven that $A / \equiv_{A}=B / \equiv_{B} \cup C / \equiv_{C}$. According to the definition of the comtrace equivalence, $A \sim_{\Theta} B C$ implies that $B \times C \subseteq$ ser. It means that for every pair of actions $b \in B$ and $c \in C$ we have $(b, c) \notin \sin$. Hence for every $a, b \in A$ we have $a \equiv_{A} b \wedge a \in B \Rightarrow b \in B$ and $a \equiv_{A} b \wedge a \in C \Rightarrow b \in C$.

It means that the graphs of the relation $\sin$ restricted to steps $B$ and $C$ not only are vertex induced parts of the graph of the relation sin restricted to the step $A$, but also are a division of this graph (i.e., the union of strongly connected components of graphs $\left.\sin \right|_{B}$ and $\left.\sin \right|_{C}$ is equal to the set of strongly connected components of the graph $\left.\sin \right|_{A}$ ), which end the proof.

Proposition 3.5. Let $\tau$ be a comtrace over $\Theta$ and $A \in \operatorname{Alph}(\tau)$. Then

$$
A / \equiv_{A} \subseteq \operatorname{Alph}(\tau) .
$$

Proof. Since $A \in A l p h(\tau)$, there exists $w, u \in \mathbb{S}$ such that $w A u \in \tau$. Applying Lemma 3.4we can construct the step sequence $A_{1} \ldots A_{n}$ composed of indivisible steps only and equivalent to step sequence consisting of $A$ only. Moreover, $A /_{\equiv_{A}}=\bigcup_{i=1 \ldots n} A_{i}$ and $w A_{1} \ldots A_{n} u \in \tau$. As a result we get that $A_{i} \in \operatorname{Alph}(\tau)$, hence $A / \equiv_{A} \subseteq \operatorname{Alph}(\tau)$.

Theorem 3.6. Let $\tau$ be a comtrace. The set indiv $(\tau)$ is a trace (with sequential semantic) over the concurrent alphabet $(\widehat{\mathbb{S}}, \widehat{\operatorname{dep}})$.

Proof. To prove the statement of the theorem it is sufficient to show two facts. Firstly, we need to prove that relation $\widehat{i n d}$ is symmetric and irreflexive. Secondly, we need to argue that by the repeated transposing of two subsequent and independent actions (in fact indivisible steps) we can reach any of other elements of the set indiv $(\tau)$ and cannot go beyond this set.

We start from the first statement. By the definition of $\operatorname{ser}$ the relation ind $=\operatorname{ser} \cap \mathrm{ser}^{-1}$ is symmetric and irreflexive. Since two indivisible steps $A$ and $B$ are in relation $\widehat{i n d}$ if all pairs of actions $(a, b) \in A \times B$ are independent, we conclude that the relation $\widehat{i n d}$ is also symmetric and irreflexive.

Let $w=u A B v$ be a step sequence from $\operatorname{indiv}(\tau)$ and $(A, B) \in \widehat{i n d}$. By the definition of the $\widehat{i n d}$ relation we have $A B \sim_{\Theta} C$ and $B A \sim_{\Theta} C$, where $C=A \cup B$. Therefore $u A B v \equiv_{\Theta} u B A v$ and the set $\operatorname{indiv}(\tau)$ is equal to its own trace closure. The last needed statement follows from Lemma 3.4 (about indivisibility of indivisible steps).

Let us suppose that there are two comtrace equivalent step sequences $u$ and $v$ belonging to $\operatorname{indiv}(\tau)$ that are not trace equivalent. Hence they differ in at least one projection to a 
binary dependent subalphabet, so there are two occurrences of indivisible steps $A$ and $B$ that appear in the two different orders and are dependent $((A, B) \in \widehat{d e p})$. Let $A$ precede $B$ in the step sequence $u$, and $B$ precede $A$ in the step sequence $v$. From the definition of comtrace equivalence there exists a sequence of equivalent step sequences $\left(w_{i}\right)_{i=1 \ldots n}$ such that $u=w_{1}, w_{i} \sim_{\Theta} w_{i+1}$, and $w_{n}=v$. In this sequence there has to exist an element $w_{i}$ where the considered occurrences of indivisible steps were for the last time in the same order as in $u\left(w_{i}=w_{i}^{\prime} X_{i} Y_{i} w_{i}^{\prime \prime}\right.$ and $w_{i+1}=w_{i+1}^{\prime} Z_{i} w_{i+1}^{\prime \prime}$ and $A \subseteq X_{i}$ and $\left.B \subseteq Y_{i}\right)$. Hence $A \times B \subseteq$ ser. Moreover, there exists an element $w_{j}$ where the considered occurrences occur for the first time after $w_{i}$ in the same order as in $v\left(w_{j}=w_{j}^{\prime} Z_{j} w_{j}^{\prime \prime}\right.$ and $w_{j+1}=w_{j+1}^{\prime} X_{j} Y_{j} w_{j+1}^{\prime \prime}$ and $A \subseteq X_{j}$ and $\left.B \subseteq Y_{j}\right)$. Hence also $B \times A \subseteq \operatorname{ser}$. Therefore $(A, B) \in \widehat{i n d}$, which gives a contradiction and completes the proof.

Proposition 3.7. Let $[w]$ be a comtrace over $\Theta$. Then

$$
\widehat{w} \in[w]
$$

and

$$
\widehat{w}=w \Leftrightarrow w \in \operatorname{indiv}(\tau) .
$$

Proof. Let $w=A_{1} \ldots A_{n}$. By the definition of the operator $\widehat{\text {, we get }}$

$$
\widehat{w}=\operatorname{minlex}\left(A_{1}\right) \ldots \text { minlex }\left(A_{n}\right) .
$$

Since minlex $\left(A_{i}\right) \equiv_{\Theta} A_{i}$ we get $\widehat{w} \equiv_{\Theta} w$, so $\widehat{w} \in[w]$.

Since $\left|\operatorname{minlex}\left(A_{i}\right)\right| \geq 1$ and $\left|\operatorname{minlex}\left(A_{i}\right)\right|=1$ if and only if $\operatorname{minlex}\left(A_{i}\right)=\widehat{\left(A_{i}\right)}=A_{i}$ we conclude that

$$
\widehat{w}=w \Leftrightarrow \forall_{i} \widehat{A_{i}}=A_{i} .
$$

By Lemma 3.4, $\widehat{\left(A_{i}\right)}=A_{i}$ if and only if $A_{i}$ is indivisible. Hence $\widehat{w}=w$ if and only if all $A_{i}$ are indivisible and

$$
\widehat{w}=w \Leftrightarrow w \in \operatorname{indiv}(\tau)
$$

As an immediate corollary of Theorem 3.6 and Proposition 3.7, we can observe that

Corollary 3.8. There is a one to one correspondence between the comtraces over comtrace alphabet $\Theta=(\Sigma$, sim, ser $)$ and traces over concurrent alphabet $\Psi=(\widehat{\mathbb{S}}, \widehat{\text { dep }})$ given by the construction of the set of indivisible steps and dependence relation on them.

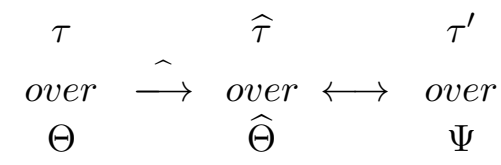

One can consider using the above correspondence to apply the methods of enumerating all traces of a given size [MPS11] to enumerate comtraces of a given size. 


\section{Projection Representation of Comtraces}

In the trace theory employing projections onto the cliques of the graph of dependence relation (see also Shi85]) turned out to be a very useful tool. We now extend this notion in the case of the binary and unary cliques only (see also [Mik08]), to define the projection representation of comtraces. In the case of traces, we have only two kinds of relationships between actions. As independent actions may be executed in any order (or together in case of step semantics) one can focus on the order implied by the dependence relation.

In the case of comtraces, the situation is more complicated. However, once more we can ignore independent actions and store information about the other three types of relations (dependency, weak dependency and strong simultaneity). Once more, it is sufficient to store the information in the form of sequences. In the case of strong simultaneity, however, we need to add a special symbol $\perp$ that separates the situations of sequential and simultaneous execution of pairs of actions being considered.

Let $a, b \in \Sigma$ and $(a, b) \notin$ ind (possibly $a=b$ ). For each such pair we define the projection function $\Pi_{a, b}^{\perp}: \mathbb{S}^{*} \rightarrow(\Sigma \cup\{\perp\})^{*}$ as follows. First, for a step $A \in \mathbb{S}$ we have

$$
\Pi_{a, b}^{\perp}(A)=\left\{\begin{array}{lll}
\epsilon & \text { for } & \{a, b\} \cap A=\varnothing \\
a & \text { for } & a \in A \wedge b \notin A \\
b a & \text { for } & \{a, b\} \subseteq A \wedge(a, b) \in w d p \\
a b & \text { for } & \{a, b\} \subseteq A \wedge(b, a) \in w d p \\
\perp & \text { for } & \{a, b\} \subseteq A \wedge(a, b) \in s s m
\end{array}\right.
$$

Note that there is a straightforward symmetry, namely for all $(a, b) \notin$ ind the equation $\Pi_{a, b}^{\perp}=\Pi_{b, a}^{\perp}$ holds. Moreover, according to the definition, we have $\Pi_{a, a}^{\perp}(A)=\epsilon$ if $a \notin A$ and $\Pi_{a, a}^{\perp}(A)=a$ if $a \in A$. Then, for a step sequence $w=A_{1} A_{2} \ldots A_{n}$ we have

$$
\Pi_{a, b}^{\perp}(w)=\Pi_{a, b}^{\perp}\left(A_{1}\right) \circ \Pi_{a, b}^{\perp}\left(A_{2}\right) \circ \ldots \circ \Pi_{a, b}^{\perp}\left(A_{n}\right) .
$$

Theorem 4.1. Let $w, u$ be step sequences over a comtrace alphabet $\Theta=(\mathbb{S}$, sim, ser $)$. Then $w \equiv_{\Theta} u \Leftrightarrow \forall_{(a, b) \notin i n d} \Pi_{a, b}^{\perp}(w)=\Pi_{a, b}^{\perp}(u)$.

Proof. $\Rightarrow$ :

We first prove that

$$
w \equiv_{\Theta} u \Rightarrow \forall_{(a, b) \notin i n d} \Pi_{a, b}^{\perp}(w)=\Pi_{a, b}^{\perp}(u) .
$$

According to the definition of comtrace equivalence, it is sufficient to prove the statement in the case of equivalent step sequences $w=A$ and $u=B C$. Let $a, b \in A$. We consider all but one of the possible relationships of these actions (the remaining case is that of independence).

Case 1: $(a, b) \in$ dep.

Since actions $a$ and $b$ occur simultaneously in the step $A$, this is impossible.

Case 2: $(a, b) \in$ ssm.

Since actions $a$ and $b$ are strongly simultaneous, Lemma 3.4 shows that they both have to occur in step $B$ or $C$. It means that

$$
\Pi_{a, b}^{\perp}(B C)=\Pi_{a, b}^{\perp}(B) \Pi_{a, b}^{\perp}(C)=\perp \epsilon=\Pi_{a, b}^{\perp}(A)
$$

or

$$
\Pi_{a, b}^{\perp}(B C)=\Pi_{a, b}^{\perp}(B) \Pi_{a, b}^{\perp}(C)=\epsilon \perp=\Pi_{a, b}^{\perp}(A) .
$$


Case 3: $(a, b) \in w d p$.

Since $B \times C \subseteq$ ser, it is impossible that $b \in C$ and $a \in B$. If they both belong to one step, we have

or

$$
\Pi_{a, b}^{\perp}(B C)=\Pi_{a, b}^{\perp}(B) \Pi_{a, b}^{\perp}(C)=(b a) \epsilon=\Pi_{a, b}^{\perp}(A)
$$

$$
\Pi_{a, b}^{\perp}(B C)=\Pi_{a, b}^{\perp}(B) \Pi_{a, b}^{\perp}(C)=\epsilon(b a)=\Pi_{a, b}^{\perp}(A)
$$

while belonging to the different steps (namely $b \in B$ and $a \in C$ ) gives

$$
\Pi_{a, b}^{\perp}(B C)=\Pi_{a, b}^{\perp}(B) \Pi_{a, b}^{\perp}(C)=b a=\Pi_{a, b}^{\perp}(A),
$$

which completes the first part of the proof.

$\Leftarrow:$

Now, let us assume that we have two step sequences $u, v \in \mathbb{S}^{*}$ and

$$
\forall(a, b) \notin i n d ~ \Pi_{a, b}^{\perp}(v)=\Pi_{a, b}^{\perp}(u) .
$$

Without loss of generality we can assume that $u=A u^{\prime}$ is in the lexicographical canonical form and $v$ consists of indivisible steps only. We claim that then there exist $v^{\prime}, v^{\prime \prime} \in \mathbb{S}^{*}$ such that $v=v^{\prime} A v^{\prime \prime}$, no action occurring in $A$ occurs in $v^{\prime}$ and $A \times a l p h\left(v^{\prime}\right) \subseteq$ ind.

Directly from the definition of the projection representation we see that all projections onto the subalphabets containing actions from the indivisible step $A$ start with the actions contained in $A$. More precisely, if $a, b \in A$ then $\Pi_{a, b}^{\perp}(u)$ starts with $a b, b a$ or $\perp$, depending on the relation between $a$ and $b$. If $a \in A$ and $b \notin A$ however, $\Pi_{a, b}^{\perp}(u)$ starts with a single action $a$.

Let $v^{\prime}$ be the longest prefix of $v$ such that $\operatorname{alph}\left(v^{\prime}\right) \cap A=\varnothing$ and $v=v^{\prime} B v^{\prime \prime}$. Obviously, all projections onto the subalphabets containing actions from the step $A$ are equal for $v$ and $B v^{\prime \prime}$. Moreover, from the definition of the indivisible step, between every two actions $a, b$ contained in $A$ there is a sequence of pairwise different actions $a=a_{1}, \ldots, a_{n}=b$ contained in $A$ such that for every $i<n$ we have $\left(a_{i+1}, a_{i}\right) \in \sin$. It means that for every such a pair of consecutive actions we have $\Pi_{a_{i}, a_{i+1}}^{\perp}(B)=a_{i} a_{i+1}$ if $\left(a_{i+1}, a_{i}\right) \in w d p$ or $\Pi_{a_{i}, a_{i+1}}^{\perp}(B)=\perp$ if $\left(a_{i+1}, a_{i}\right) \in \mathrm{ssm}$. Nevertheless, if $a_{i+1}$ is in $B$ then also $a_{i}$ have to be in $B$. Otherwise $\Pi_{a_{i}, a_{i+1}}^{\perp}(B)$ would start with $a_{i+1}$. This proves that, since $A \cap B \neq \varnothing, A \subseteq B$. Using similar arguments, we can see that since $B$ is indivisible, no other action may occur in $B$ and $A=B$.

It remains to be shown that $A \times \operatorname{alph}\left(v^{\prime}\right) \subseteq$ ind. Let $a \in A$ and $c \in \operatorname{alph}\left(v^{\prime}\right)$. Clearly, $c \notin A$ from the definition of sequence $v^{\prime}$. In the step sequence $v$ the action $c$ appears before action $a$ so, if they are not independent, $\Pi_{a, c}^{\perp}(v)=\Pi_{a, c}^{\perp}(u)$ starts with $c$. But $a \in A$ and $c \notin A$, and so $\Pi_{a, c}^{\perp}(u)$ starts with $a$. This contradicts our assumption that $a$ and $c$ are not independent and proves that $v \equiv_{\Theta} A v^{\prime} v^{\prime \prime}$. Repeating the above reasoning, we obtain that $u$ is the lexicographical canonical form of $v$ which ends the second part of the proof.

The projection representation of a comtrace $\tau$ is a function $\Pi_{\tau}^{\perp}:(\Sigma \times \Sigma) \backslash$ ind $\rightarrow(\Sigma \cup\{\perp\})^{*}$, given by $\Pi_{\tau}^{\perp}(a, b)=\Pi_{a, b}^{\perp}(\tau)$. Moreover, any function $\Pi^{\perp}:(\Sigma \times \Sigma) \backslash$ ind $\rightarrow(\Sigma \cup\{\perp\})^{*}$ is called a projection set. Clearly, not every projection set is a projection representation of a comtrace. In the next section, we give a procedure that decides whether a given projection set is a projection representation of a comtrace. Moreover, if the answer is positive, the procedure computes a representative of such a comtrace. 
First, however, we provide the algorithm computing projection representation of a comtrace. This algorithm comes directly from the definition. However, to say anything about the time complexity of the algorithm, it is important to discuss the data structures which might be used by this algorithm. At the beginning, let us consider the input. We get a comtrace alphabet $\Theta$ which consists of the alphabet $\Sigma$ of size $k$ and two relations, sim and ser, of size at most $k^{2}$ each. We also get a step sequence $w$ which steps consist of $n$ occurrences of atomic actions (elements of $\Sigma$ ) all together. As a result, we obtain the set of at most $k^{2}$ sequences (projections onto specified subalphabets).

We process the step sequence $w$ step by step, which means that the algorithm is online (i.e. during the computation we achieve correct results for each proper prefix of $w$ ). The processing of a single step is done according to the definition of projections onto the pairs in the specified relation. It is worth carrying out some preprocessing and, for every action, compute the list of all subalphabets in which it may occur. By storing, for every computed projection, the number of the step when it was most recently updated, we avoid problems with the special cases of relations $w d p$ and ssm (in these cases two rather than one action may be added to one sequence while processing a single step).

Proposition 4.2. The procedure of computing $\Pi_{\tau}^{\perp}$ from a step sequence $w \in \tau$ has the time and memory complexity of $O(n k)$.

Proof. The proof is straightforward. The algorithm is naturally divided into $n$ stages grouped by steps of input step sequence. In each stage we process a single action and add it to at most $k$ sequences updating at most $k$ counters. Hence each stage can be done in the time linearly proportional to the size of the alphabet. Therefore whole procedure has the time complexity of $O(n k)$.

Theorem 4.3. Testing comtrace equivalence can be done in the time complexity of $O(n k)$.

Proof. Notice that the output of procedure discussed in Proposition 4.2 has also memory complexity of $O(n k)$. Hence for two step sequences we can compute their projection representations and compare them sequence by sequence.

4.1. Reconstructing Step Sequence from Projection Set. The idea of constructing a step sequence from a projection set is based on revealing the first possible step whose projection representation would form a set of prefixes of a given projection set. At first, we identify the set of all possible elements of such a step. We do it in two stages. We first identify the set of conditionally possible actions, i.e. those actions whose first occurrences are the first (or in particular situations the second) actions in all projections, where they could appear. Note that we treat the special symbol $\perp$ as a pair of proper actions, so its occurrence means that both actions might be conditionally possible. After this identification, we remove actions that cannot satisfy some of the necessary conditions. These conditions are related to the cases when the considered action appears as the second action in some sequences connected with the weak dependence relation or are verified positively because of the special symbol $\perp$.

As a result of the first stage, we obtain the set of all actions that may appear in the first step of the constructed sequence. The second stage consists of dividing this set into indivisible steps and combining those indivisible steps into one of the allowed steps. The result is obtained by taking advantage of the weak dependence relation inside the set of 
indivisible steps. It is similar to the ideas behind the proof of Lemma 3.4. Let us look into the details of the proposed procedure.

Recall that by $\operatorname{pref}_{k}(w)=a_{1} \ldots a_{k}$ we denote the k-prefix of $w$. Let $\Pi^{\perp}$ be a projection set. We say that an action $a \in \Sigma$ is conditionally possible for projection set $\Pi^{\perp}$ if and only if for all $b \in \Sigma$ the following implications are satisfied:

- $(a, b) \in d e p \Rightarrow \operatorname{pref}_{1}\left(\Pi^{\perp}(a, b)\right)=a$

- $(b, a) \in w d p \Rightarrow \operatorname{pref}_{1}\left(\Pi^{\perp}(a, b)\right)=a$

- $(a, b) \in w d p \Rightarrow \operatorname{pref}_{1}\left(\Pi^{\perp}(a, b)\right)=a \vee \operatorname{pref}_{2}\left(\Pi^{\perp}(a, b)\right)=b a$

- $(a, b) \in s s m \Rightarrow \operatorname{pref}_{1}\left(\Pi^{\perp}(a, b)\right)=a \vee \operatorname{pref}_{1}\left(\Pi^{\perp}(a, b)\right)=\perp$

We denote all conditionally possible actions as cpa and define the relation $c n d \subseteq \Sigma \times \Sigma$, which describes the conditions that must be satisfied. Only in situations where

$$
(a, b) \in w d p \wedge \operatorname{pref}_{2}\left(\Pi^{\perp}(a, b)\right)=b a
$$

or

$$
(a, b) \in \operatorname{ssm} \wedge \operatorname{pref}_{1}\left(\Pi^{\perp}(a, b)\right)=\perp
$$

we say that the existence of action $b$ in the constructed step is a necessary condition for the presence of action $a$ in this step, which is denoted by $(a, b) \in c n d$.

We exclude conditionally possible actions with conditions impossible to satisfy to form the set of possible actions. Any action $a \in \Sigma$ that is not conditionally possible in $\Pi^{\perp}$ is impossible in $\Pi^{\perp}$. Moreover, any action $a$ conditionally possible under impossible condition (i.e. $(a, b) \in$ cnd and $b$ is impossible) is also impossible. Formally, the set of impossible actions for the projection function $\Pi^{\perp}$ is the smallest set imp that satisfies the following conditions:

- $\Sigma \backslash c p a \subseteq i m p$

- $b \in i m p \wedge(a, b) \in c n d \Rightarrow a \in i m p$

Let $M\left(\Pi^{\perp}\right)$ be the set of actions which are not impossible (which means that they are possible) for projection set $\Pi^{\perp}$. The next operation is to choose a subset of $M\left(\Pi^{\perp}\right)$ which could be a first step of the reconstructed step sequence. To do so we take a sequential trace over $\widehat{\mathbb{S}}$, given by the step sequence $\widehat{M\left(\Pi^{\perp}\right)}$ (see Corollary 3.8$)$. Note that for any $a \in M\left(\Pi^{\perp}\right.$ ) we have $\#_{a}\left(\widehat{M\left(\Pi^{\perp}\right)}\right) \leq 1$. We take any nonempty trace prefix $B_{1} \ldots B_{n}$ of step sequence $\widehat{M\left(\Pi^{\perp}\right)}$ and set $B=\bigcup_{i} B_{i}$ as a requested step. The procedure just described is justified by the following facts:

Proposition 4.4. Let $\Pi^{\perp}$ be a projection set over a comtrace alphabet $\Theta$ and $B \subseteq M\left(\Pi^{\perp}\right)$ a set of actions constructed according to the procedure described above.

If $b \in B$ and $a \in M\left(\Pi^{\perp}\right)$ then

$$
(b, a) \in \sin ^{*} \Longrightarrow[a]_{\equiv_{M\left(\Pi^{\perp}\right)} \subseteq B .}
$$

Proof. Let $\widehat{M\left(\Pi^{\perp}\right)}=B_{1} \ldots B_{n}$, where all the $B_{m}$ 's are indivisible. Since $a, b \in M\left(\Pi^{\perp}\right)$ there exist $1 \leq p, q \leq n$ such that $a \in B_{p}$ and $b \in B_{q}$. By Lemma $3.4[a]_{\equiv_{M\left(\Pi^{\perp}\right)}}=B_{p}$. By Corollary 3.8, $\widehat{M\left(\Pi^{\perp}\right)}$ forms a sequential trace over $\widehat{\mathbb{S}}$. In the above procedure we use one of trace prefixes of $\widehat{M\left(\Pi^{\perp}\right)}$, taking $B$ as the union of all indivisible steps (actions of $\widehat{\mathbb{S}}$ ) contained in this prefix. Hence $B_{q} \subseteq B$. If $p=q$ we have that $B_{p}=B_{q}$ and $B_{p}=[a]_{\equiv_{M\left(\Pi^{\perp}\right)}} \subseteq B$. Let 
us consider the case $B_{p} \neq B_{q}$. It is sufficient to prove that $B_{p}$ occurs before $B_{q}$ in all trace prefixes of $\widehat{M\left(\Pi^{\perp}\right)}$.

Since $(b, a) \in \sin ^{*}$, there exists a sequence of actions $b=c_{1} \ldots c_{k}=a$ such that $\left(c_{i}, c_{i+1}\right) \in \sin$ for every $0<i<k$. Hence there exists a sequence of steps $u=C_{1} \ldots C_{k}$ such that $c_{i} \in C_{i}$. Clearly, $C_{i}$ might be equal to $C_{i+1}$, for some $0<i<k$, but surely $C_{1} \neq C_{k}$. However, for distinct $i, j$ we have $\left(C_{i}, C_{j}\right) \in \widehat{d e p}$. Moreover, each $C_{i}$ is contained in $\operatorname{Alph}\left(M\left(\Pi^{\perp}\right)\right)$ and if $C_{i}$ occurs before $C_{j}$ in $u$, then it also has to occur before $C_{j}$ in $\widehat{M\left(\Pi^{\perp}\right)}$.

If $C_{i}$ and $C_{i+1}$ are different, then during the division of the step $\widehat{M\left(\Pi^{\perp}\right)}$ (see Lemma 3.4 ) they have to get to different parts (like steps $B$ and $C$ in Lemma 3.4). Since $\left(c_{i}, c_{i+1}\right) \in \sin$, it is impossible to have $C_{i} \times C_{i+1} \subseteq$ ser. This shows that their orders of occurring in $u$ and $\widehat{M\left(\Pi^{\perp}\right)}$ are reversed. Moreover, this remains true for every sequence over $\widehat{\mathbb{S}}$ equivalent to $\widehat{M\left(\Pi^{\perp}\right)}$. Finally, we conclude that what we have shown applies not only to consecutive and distinct steps of $u$ but also to all its distinct elements, including $C_{1}=B_{q}$ and $C_{k}=B_{p}$, which end the proof.

Theorem 4.5. Let $w=A_{1} \ldots A_{n}$ be a step sequence, and $\Pi^{\perp}$ be the projection representation of $[w]$. Then

$$
A_{1} \subseteq M\left(\Pi^{\perp}\right) .
$$

Proof. Since $\Pi^{\perp}$ is the projection representation of $[w]$, for all $(a, b) \notin$ ind we have

$$
\Pi^{\perp}(a, b)=\Pi_{a, b}^{\perp}(w)=\Pi_{a, b}^{\perp}\left(A_{1} \ldots A_{n}\right) .
$$

Hence all actions contained in $A_{1}$ are conditionally possible. Moreover, $(a, b) \in$ cnd means that $(a, b) \in w d p$ or $(a, b) \in s s m$. In the first case, $\operatorname{pref}_{2}\left(\Pi^{\perp}(a, b)\right)=b a$, so $b \in A_{1}$. Similarly, if $(a, b) \in$ ssm then $\operatorname{pref}_{2}\left(\Pi^{\perp}(a, b)\right)=\perp$, so $b \in A_{1}$.

Since $(a, b) \in$ cnd and $a \in A_{1}$ implies $b \in A_{1}$, and $A_{1} \subseteq c p a$, we conclude that $A_{1} \cap i m p=\varnothing$. This proves that $A_{1} \subseteq M\left(\Pi^{\perp}\right)$.

As a result, we can extract step $B$ from $\Pi^{\perp}$. The extraction function

$$
\text { extr }:\left((\Sigma \times \Sigma \backslash \text { ind })^{*} \rightarrow(\Sigma \cup \perp)^{*}\right) \times \mathbb{S} \rightarrow\left((\Sigma \times \Sigma \backslash \text { ind })^{*} \rightarrow(\Sigma \cup \perp)^{*}\right)
$$

for projection set $\Pi^{\perp}$ and set $B \subseteq M\left(\Pi^{\perp}\right)$ constructed using the procedure described above is defined as:

$$
\operatorname{extr}\left(\Pi^{\perp}, B\right)(a, b)=\left\{\begin{array}{lll}
\Pi^{\perp}(a, b) & \text { for } & |\{a, b\} \cap B|=0 \\
\operatorname{suff}_{2}\left(\Pi^{\perp}(a, b)\right) & \text { for } & |\{a, b\} \cap B|=1 \\
\operatorname{suff}_{2}\left(\Pi^{\perp}(a, b)\right) & \text { for } & |\{a, b\} \cap B|=2 \wedge(a, b) \in \text { ssm } \\
\operatorname{suff}_{3}\left(\Pi^{\perp}(a, b)\right) & \text { for } & |\{a, b\} \cap B|=2 \wedge(a, b) \in w d p \cup w d p^{-1}
\end{array}\right.
$$

Example 4.6. Let us consider the comtrace $\tau$ from Example 2.13,

The projection representation of $\tau$ (omitting projections to the unary subalphabets), grouped by the types of relation between the elements of subalphabets on which we project are:

$$
\begin{array}{lll}
\text { dep : } & \Pi_{\tau}^{\perp}(b, d)=d b & \Pi_{\tau}^{\perp}(c, d)=d \\
\text { ssm : } & \Pi_{\tau}^{\perp}(a, c)=a & \\
w d p: & \Pi_{\tau}^{\perp}(c, b)=b & \Pi_{\tau}^{\perp}(d, a)=d a
\end{array}
$$


The set of conditionally possible actions for $\Pi_{\tau}^{\perp}$ is $\{a, d\}$, while $(a, d) \in$ cnd. Every conditionally possible action is also possible, and so $M\left(\Pi_{\tau}^{\perp}\right)=\{a, d\}$. This gives the set of two indivisible steps $(a)$ and $(d)$ and, finally, two steps that may appear as the first step of the constructed sequence: $(d)$ and $(a d)$.

Theorem 4.7. Let $\Pi_{\tau}^{\perp}$ be the projection representation of a comtrace $\tau$, and $M\left(\Pi^{\perp}\right)$ be a maximal possible step of $\Pi_{\tau}^{\perp}$. For every allowed set $B \in \mathbb{S}$, we have

$$
\tau=B \circ \sigma, \text { where } \Pi_{\sigma}^{\perp}=\operatorname{extr}\left(\Pi_{\tau}^{\perp}, B\right) .
$$

Proof. By the Theorem 4.1 it is sufficient to prove that $\Pi_{\tau}^{\perp}=\Pi_{B \circ \sigma}^{\perp}$. In other words, we have to show that for all $(a, b) \notin i n d$, we have $\Pi_{\tau}^{\perp}(a, b)=\Pi_{B}^{\perp}(a, b) \circ \Pi_{\sigma}^{\perp}(a, b)$.

The proof can be split in a natural way into three parts, depending on the type of relation between the actions being considered. Let us examine the projections onto $(a, b) \in \operatorname{dep}$. We have $\Pi_{B}^{\perp}(a, b)$ that is equal to the first action of $\Pi_{\tau}^{\perp}(a, b)$ if $|B \cap\{a, b\}|=1$, and to $\epsilon$ otherwise. In both cases $\Pi_{B}^{\perp}(a, b) \circ \Pi_{\sigma}^{\perp}(a, b)=\Pi_{\tau}^{\perp}(a, b)$.

Almost the same proof works for the remaining two cases, when $(a, b) \in w d p$ or $(a, b) \in$ ssm.

By suitably using the extraction function, we can compute any representative of a comtrace $\tau$. In particular, similarly to the case of canonical forms, we can do this using a maximal or minimal strategy. In the maximal strategy, we always take the whole set $M\left(\Pi^{\perp}\right)$ and, as a result, we obtain Foata canonical form of the original comtrace. In the minimal strategy, we take the first step of the step sequence $\widehat{M\left(\Pi^{\perp}\right)}$ and obtain the lexicographical canonical form.

The algorithm reconstructing a step sequence from a projection representation of a comtrace follows the notions defined above. From the technical point of view, some concrete decisions concerning data structures are worth noticing. The whole algorithm can be divided into stages. In each stage we compute a set of allowed steps, choose one, and extract it from the projection set. The procedure is repeated until a projection set $\Pi_{i}^{\perp}$ or computed set $M\left(\Pi_{i}^{\perp}\right)$ become empty. In the first case, it returns a step sequence consisting of $n$ occurrences of actions. In the second case, the algorithm returns that an input is not a projection representation of a comtrace.

A single stage starts from computing the set of conditionally possible actions and the relation cnd describing the conditions. A good idea is to preprocess, for every action, a list of pointers which helps to investigate only the projections related to this action. Doing so, we can check conditional possibility in the time linearly dependent on the size of alphabet, denoted by $k$. Simultaneously, we build the directed graph of conditions. In the time linearly dependent on the number of arcs in this graph, we remove from the set of conditionally possible actions all impossible ones (browsing, using DFS, all paths which begin in vertices which are not conditionally possible).

In the next phase, we compute a vertex induced subgraph of the sin relation that contains all possible actions and, once more using DFS, we compute a graph of its strongly connected components (called condensation graph [Deo74]). The condensation graph is an acyclic directed graph of the partial order of the sequential trace associated with $\widehat{M\left(\Pi^{\perp}\right)}$. We choose an arbitrary upper set of the condensation graph, that corresponds to the trace prefix of $\widehat{M\left(\Pi^{\perp}\right)}$. To obtain Foata canonical form, we take the maximal upper set by choosing the whole condensation graph. If we wish to obtain the lexicographical canonical 
form, we should choose the $\widehat{\leq}$-smallest allowed step. To compute it, we may consider only the maximal elements of provided condensation graph. They correspond to the elements of $\widehat{\mathbb{S}}$ which may be placed at the first positions in the sequential trace $\widehat{M\left(\Pi^{\perp}\right)}$.

In the last phase, we need to extract the chosen allowed step. We do it according to the definition of the extraction operation. During this phase, we can once more use the precomputed lists of pointers.

Proposition 4.8. Projection set $\Pi^{\perp}$ is the projection representation of a comtrace if and only if the procedure described above ends with the empty projection set.

Proof. We give the proof only for the case when the maximal strategy is used. Note that the input data is finite and the procedure stops when the set $M\left(\Pi^{\perp}\right)$ is empty for the remaining set of words. From Theorem 4.7 we deduce that if the remaining projection set is empty then the input is the projection representation of the constructed comtrace. Suppose that we have nonempty projection set $\Pi^{\perp}$ that is a projection representation of comtrace $\tau$ and empty set of allowed actions. Let us consider an arbitrary step sequence $u=A_{1} \ldots A_{n}$ that is contained in $\tau$, and an arbitrary action $a$ contained in $A_{1}$. Then, by the definition of projection representation, the action $a$ has to be possibly allowed. This proves that $A_{1} \subseteq c p a$. Moreover, since in any projection before, or simultaneously with, a may occur only other action from the step $A_{1}$, if the existence of action $b$ is a necessary condition for the presence of action $a$ (i.e. $(a, b) \in c n d$ ), then $b$ is also an element of $A_{1}$. Therefore, none of the actions from step $A_{1}$ is impossible, which contradicts the emptiness of the set of allowed actions and ends the proof.

Theorem 4.9. The procedure of computing canonical forms from a projection representation of a comtrace has the time complexity of $O\left(n k^{2}\right)$.

Proof. The procedure consist of at most $n$ stages. In each part, we carry out some operations on at most $k^{2}$ lists and graph of size $k^{2}$. All graph operations, including computing the compensation graph and choosing minimal or maximal upper set are linear in the size of graph. This gives an overall time complexity of $O\left(n k^{2}\right)$.

4.2. Traces as a subclass of comtraces. In Section 1 we defined EN-systems as a special case of ENI-systems without inhibitors and with the sequential semantics. We also introduced traces as a model of the causal behaviour of EN-systems. In this section, we show what kind of comtraces are directly related to systems without inhibitors.

A comtrace alphabet $\Theta=(\Sigma$, sim, ser $)$ with the empty relation sin is called radical comtrace alphabet. Moreover, comtraces over this alphabet are called radical comtraces. The radicalism of such comtraces means that the actions may be only dependent or independent, hence they behave exactly like step traces. Later in this section we discuss some properties of this subclass.

Proposition 4.10. Let $\tau \in \mathbb{S}^{*}$ be a radical comtrace and $w \in \operatorname{indiv}(\tau)$. Then each step of $w$ is a singleton.

Proof. The proof is straightforward. Notice that since the relation sin is empty, every action $a$ of every step $A \in \mathbb{S}$ forms an indivisible step. Hence all indivisible steps are singletons, which ends the proof. 
Corollary 4.11. Let $\Theta=(\Sigma$, sim, ser $)$ be a radical comtrace alphabet. Then

$$
\operatorname{lex}(\widehat{\mathbb{S}})=\Sigma \text {. }
$$

Note that since the relation sin is empty and all steps are singletons, for all steps $A, B \in \widehat{\mathbb{S}}$ we have $(A, B) \in \widehat{d e p}$ if and only if $(\operatorname{lex}(A), \operatorname{lex}(B)) \in \operatorname{dep}$.

Using Theorem 3.6 and Lemma 4.11 we can associate an alphabet of indivisible steps $\widehat{\mathbb{S}}$ with $\Sigma$ and radical comtrace $\tau$ over a comtrace alphabet $\Theta=(\Sigma$, sim, ser $)$ with a step trace $\sigma$ over the concurrent alphabet $\Psi=(\Sigma$,ind). We say that such a step trace $\sigma$ is a trace representation of a radical comtrace $\tau$. The following facts show this correspondence in details.

Proposition 4.12. Let $\Theta=(\Sigma$, sim, ser $)$ be a radical comtrace alphabet. Then a set $A \subseteq \Sigma$ is a step in $\Theta$ if and only if $A$ is a step in $\Psi=(\Sigma, \widehat{d e p})$.

Proof. It is sufficient to prove that $\operatorname{sim}=i n d$.

Indeed, since sin is empty, we have $\operatorname{sim} \backslash$ ser $=\varnothing$, hence by ser $\subseteq$ sim we get $\operatorname{sim}=$ ser. Recall that sim is symmetric, and so is ser. By the definition of relations in comtraces,

$$
\text { ind }=\operatorname{ser} \cap \operatorname{ser}^{-1}=\operatorname{ser}=\operatorname{sim} .
$$

Theorem 4.13. Let $\tau$ be a radical comtrace and $\sigma$ be its step trace representation. Then $\Pi_{\tau}^{\perp}=\Pi_{\sigma}$.

Proof. Let $\tau=\left[A_{1} \ldots A_{n}\right]=\sigma$. The relation sin is empty, so in the case of comtraces we consider only projections to pair of actions that are dependent. As a result, we conclude that the projections on the same pairs of actions are the same, no matter whether we consider comtraces or step traces, $\Pi_{a, b}^{\perp}(\tau)=\Pi_{a, b}(\sigma)$ for every $(a, b) \in$ dep, hence $\Pi_{\tau}^{\perp}=\Pi_{\sigma}$.

Corollary 4.14. Let $\tau \in \mathbb{S}^{*}$ be a radical comtrace and $\sigma$ be its step trace representation. Their canonical forms (both lexicographical and Foata) are equal.

Corollary 4.15. The correspondence between comtraces over $\Theta=(\Sigma$, sim, ser $)$ and traces over $\Psi=(\widehat{\mathbb{S}}, \widehat{d e p})$ (see Corollary [3.8) collapses in case of radical comtraces to

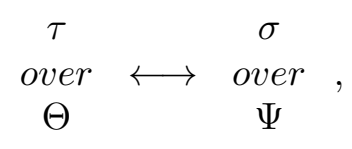

where $\tau=\sigma$ as sets of step sequences.

\section{Summary AND FUTURE WORK}

In this paper we presented a number of algebraic aspects of combined traces. Similar algebraic tools were successfully used in the study of the Mazurkiewicz traces, a simpler model for capturing and analysing concurrent behaviours.

In particular, we defined lexicographical canonical form of a comtrace and its projection representation. We gave two simple algorithms which generate these representations from arbitrary step sequence. Those algorithms seem to have the potential to provide a base for the development of solutions to some natural problems related to the comtrace theory, like 
model verification [EH08, RSK13]. In particular, one can use them to design efficient methods for the enumeration of all the representatives of a fixed comtrace, and the enumeration of all comtraces of a given size.

Another interesting direction of further studies would be the notion of recognisable and rational languages of combined traces. The projection representation seems to be a good starting point in this area; in particular, if one recalls Zielonka's asynchronous automata [Zie87] for traces. Finally, the projection representation may find an application in another important aspect of combined trace theory. A fair strategy of reconstructing step sequences from a projection set might be useful as a starting point in the theory of infinite combined traces.

Acknowledgments. I would like to thank Maciej Koutny and anonymous reviewers for their constructive comments, which helped to improve this paper.

This research was supported by a fellowship funded by the "Enhancing Educational Potential of Nicolaus Copernicus University in the Disciplines of Mathematical and Natural Sciences" Project POKL.04.01.01-00-081/10.

\section{REFERENCES}

[CF69] P. Cartier and D. Foata. Problèmes Combinatoires de Commutation et Réarrangements, volume 85 of LNM. Springer, Berlin, 1969.

[Deo74] N. Deo. Graph theory with applications to engineering and computer science. Prentice-Hall, 1974.

[DM97] V. Diekert and Y. Métivier. Partial commutation and traces. In Handbook of Formal Languages, volume 3, pages 457-533. Springer, 1997.

[DR95] V. Diekert and G. Rozenberg, editors. The Book of Traces. World Scientific, Singapore, 1995.

[EH08] J. Esparza and K. Heljanko. Unfoldings: A Partial-Order Approach to Model Checking. Monographs in Theoretical Computer Science. 2008.

[JK95] R. Janicki and M. Koutny. Semantics of inhibitor nets. Information and Computation, 123(1):1-16, 1995.

[JKK11] R. Janicki, J. Klein, and M. Koutny. Quotient monoids and concurrent behaviours. In Carlos Martín-Vide, editor, Scientific Applications of Language Methods, chapter 6, pages 313-386. Imperial College Press, London, 2011.

[JL11] R. Janicki and D. T. M. Le. Modelling concurrency with comtraces and generalized comtraces. Information and Computation, 209(11):1355-1389, 2011.

[Maz77] A. Mazurkiewicz. Concurrent program schemes and their interpretations. Daimi report pb-78, Aarhus University, 1977.

[Mik08] Ł. Mikulski. Projection representation of Mazurkiewicz traces. Fundamenta Informaticae, 85:399408, 2008.

[MK11] Ł. Mikulski and M. Koutny. Hasse diagrams of combined traces. Technical report cs-tr-1301, Newcastle University, 2011.

[MPS11] Ł. Mikulski, M. Piątkowski, and S. Smyczyński. Algorithmics of posets generated by words over partially commutative alphabets. In Jan Holub and Jan Žd'árek, editors, Proceedings of the Prague Stringology Conference 2011, pages 209-219, Czech Technical University in Prague, Czech Republic, 2011.

[RSK13] C. Rodríguez, S. Schwoon, and V. Khomenko. Contextual merged processes. In José-Manuel Colom and Jörg Desel, editors, Proceedings of the 34th International Conference on Applications and Theory of Petri Nets (ICATPN'13), volume 7927 of Lecture Notes in Computer Science, pages 29-48. Springer, 2013.

[Shi85] M. W. Shields. Concurrent machines. The Computer Journal, 28(5):449-465, 1985.

[Vog91] W. Vogler. A generalization of traces. ITA, 25:147-156, 1991.

[Zie87] W. Zielonka. Notes on finite asynchronous automata. RAIRO, 21:99-135, 1987.

This work is licensed under the Creative Commons Attribution-NoDerivs License. To view a copy of this license, visit http://creativecommons.org/licenses/by-nd/2.0/ or send a letter to Creative Commons, 171 Second St, Suite 300, San Francisco, CA 94105, USA, or Eisenacher Strasse 2, 10777 Berlin, Germany 